\title{
The Bidirectional Optimization of Carbon Fiber Production by Neural Network with a GA-IPSO Hybrid Algorithm
}

\author{
Jiajia Chen, ${ }^{1}$ Yongsheng Ding, ${ }^{1,2}$ and Kuangrong Hao ${ }^{1,2}$ \\ ${ }^{1}$ College of Information Sciences and Technology, Donghua University, Shanghai 201620, China \\ ${ }^{2}$ Engineering Research Center of Digitized Textile \& Fashion Technology, Ministry of Education, \\ Donghua University, Shanghai 201620, China
}

Correspondence should be addressed to Yongsheng Ding; ysding@dhu.edu.cn

Received 10 January 2013; Accepted 24 January 2013

Academic Editor: Yang Tang

Copyright ( 92013 Jiajia Chen et al. This is an open access article distributed under the Creative Commons Attribution License, which permits unrestricted use, distribution, and reproduction in any medium, provided the original work is properly cited.

\begin{abstract}
A hybrid approach of genetic algorithm (GA) and improved particle swarm optimization (IPSO) is proposed to construct the radial basis function neural network (RNN) for real-time optimizing of the carbon fiber manufacture process. For the three-layer RNN, we adopt the nearest neighbor-clustering algorithm to determine the neurons number of the hidden layer. When the appropriate network structure is fixed, we present the GA-IPSO algorithm to tune the parameters of the network, which means the center and the width of the node in the hidden layer and the weight of output layer. We introduce a penalty factor to adjust the velocity and position of the particles to expedite convergence of the PSO. The GA is used to mutate the particles to escape local optimum. Then we employ this network to develop the bidirectional optimization model: in one direction, we take production parameters as input and properties indices as output; in this case, the model is a carbon fiber product performance prediction system; in the other direction, we take properties indices as input and production parameters as output, and at this situation, the model is a production scheme design tool for novel style carbon fiber. Based on the experimental data, the proposed model is compared to the conventional RBF network and basic PSO method; the research results show its validity and the advantages in dealing with optimization problems.
\end{abstract}

\section{Introduction}

Carbon fiber is a novel breed of ideal engineering materials which has high strength and modulus; hence, it has great influence on aspects of military, industry, economy, and so on. Due to its high strength, high break strength, high modulus, small diameter, low density, maximum crystallinity, and low comonomer contents, polyacrylonitrile-(PAN-) based carbon fiber dominates the market and the optimization of its manufacture process receives increasing attention from scholars and researchers in recent years [1]. Most research groups have been working on the post spinning process, which means improving the properties of carbon fiber by gas adsorption application or water treatments [2]. Liu et al. [3] investigated electrochemical oxidation treatment of PANbased carbon fibers by some instruments such as scanning electron microscopy (SEM) and X-ray diffraction (XRD), gave out the guidance when properties interfacial bonding strength and tensile strength were improved simultaneously.
Wang and Yin [4] discussed how the chemical element potassium permanganate $\left(\mathrm{KMnO}_{4}\right)$ modified properties of PAN fibers during heat treatment process by lab instruments. Rahman et al. [5] investigated the effects of residence time in the solvent-free coagulation bath on the formation and mechanical properties of PAN fibers. Liang et al. [6] proposed a bioinspired multilayered intelligent cooperative controller for stretching process of fiber production. From the above, we know that they always analyze properties by drawing support from physical or chemical instruments [79], consider relationship between production parameters and fiber properties rarely. On one hand, researchers in materials science are based on different entry point with engineering scholars, which leads to this situation. On the other hand, the production process of carbon fiber is a complex system, it contains the following steps: dissolution, computation, off single deaeration, filtration, spinneret, coagulating bath, washing, apply oil, drying, high temperature draft, heat setting, drying, and winding. These procedures can be viewed as 


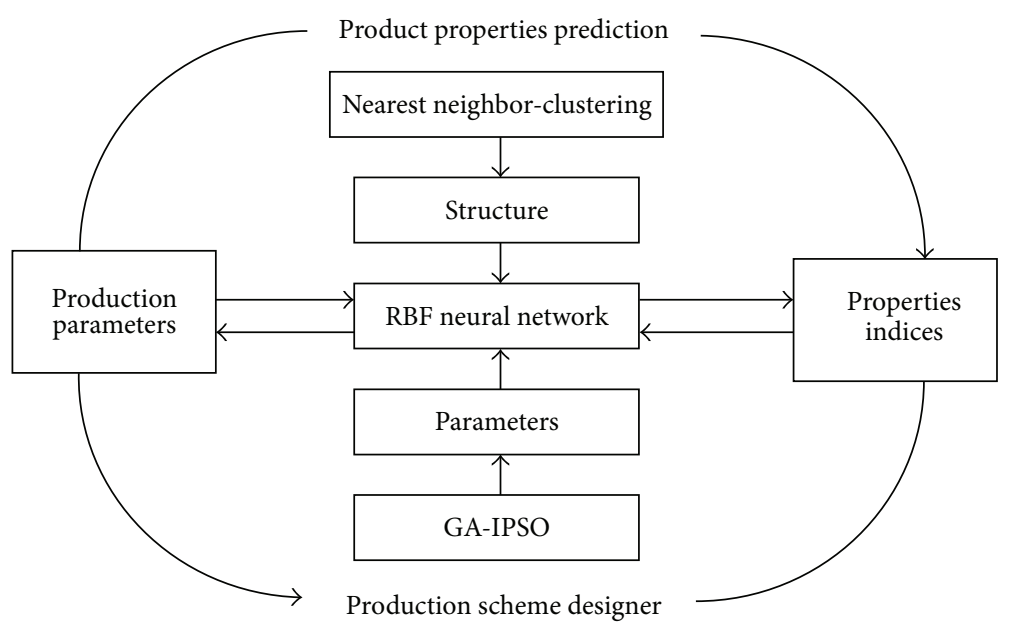

FIgURE 1: The bidirectional optimization model of carbon fiber production process.

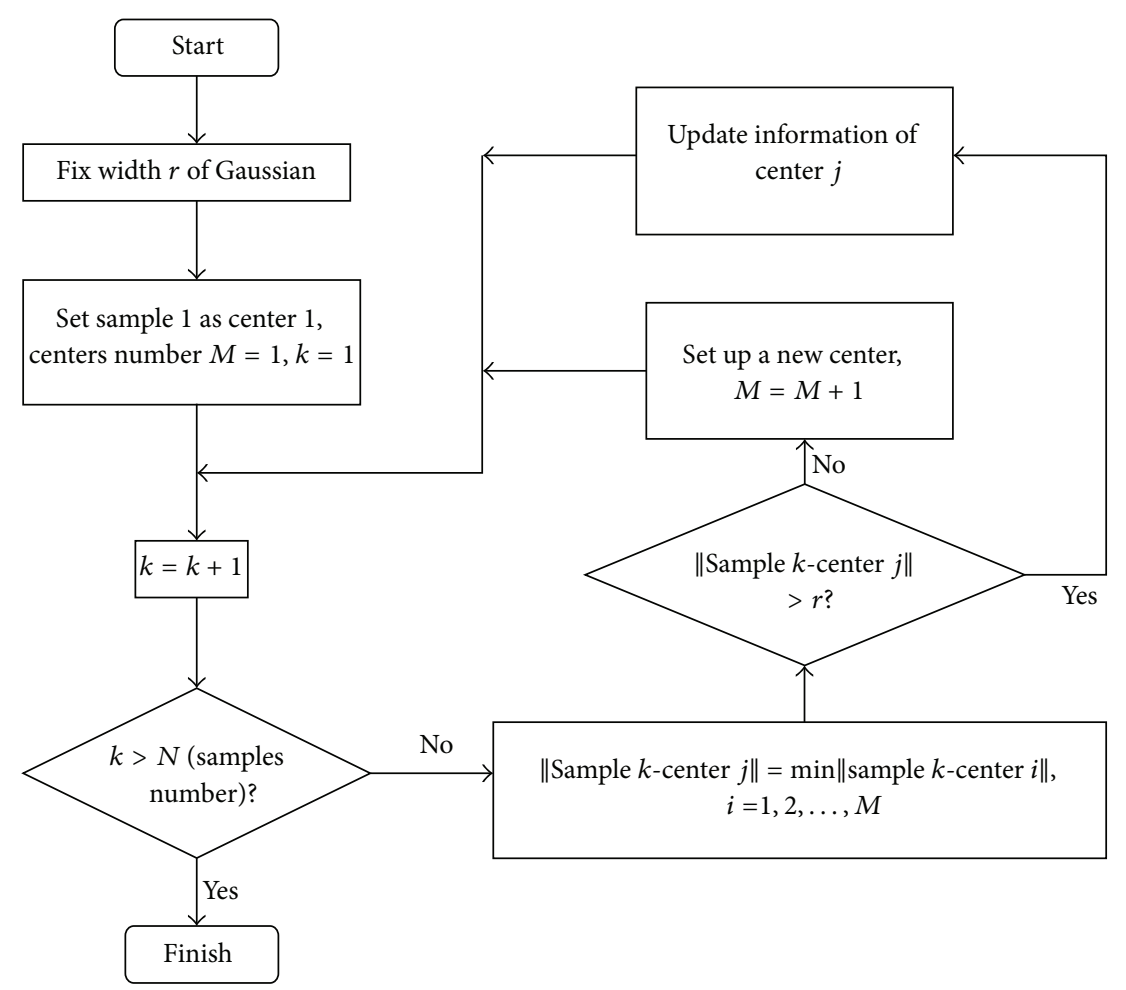

FIGURE 2: The nearest neighbor-clustering algorithm.

subsystems, every of which has its own control factors. These factors affect and restrict the whole system's development and evolution, whereas they are not only influenced by external environment but also by the internal coupling phenomenon between subsystems. Consequently, it is difficult to build a mathematical model to describe the relationship between production parameters and properties indices linearly, or just connect the relational expressions of every subsystem simply.

With spring up of computational intelligent methods, they have provided a powerful tool to approximate the functional form of an unknown complex nonlinear system. Among them, since its extremely strong adaptive capabilities in response to nonlinear behaviors, artificial neural network (ANN) have been presented to deal with modeling dynamic process for product and process design, monitoring, and control. Kadi [10] adopted the ANN to estimate the performance of the fiber-reinforced composites. Yu et al. [11] introduced a novel ANN to conduct the fabric selection among different fabric specimen with a reasonable rule set. However, topology of ANN is not compact enough which leads to more training time for learning. Then radial basis function (RBF) neural network (RNN) rises in artificial intelligence filed. Some researchers investigated RNN from aspects of center selection, learning theory, boundary value 


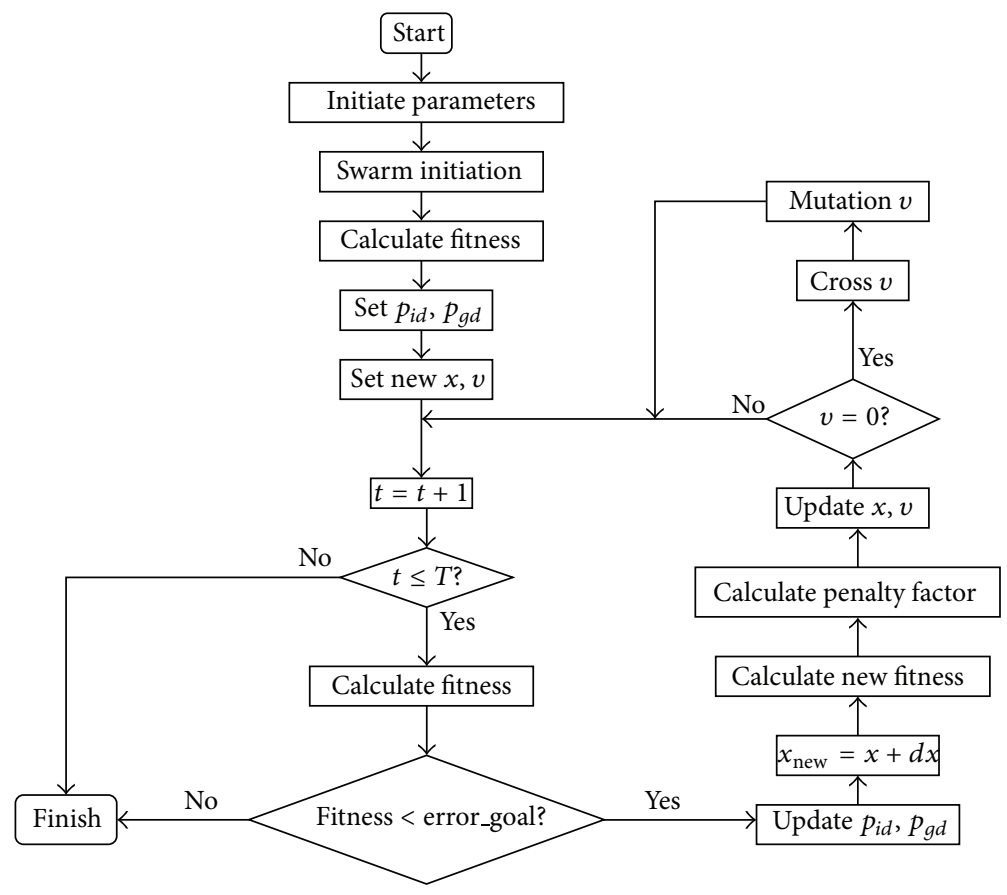

FIgURE 3: The flowchart of GA-IPSO.

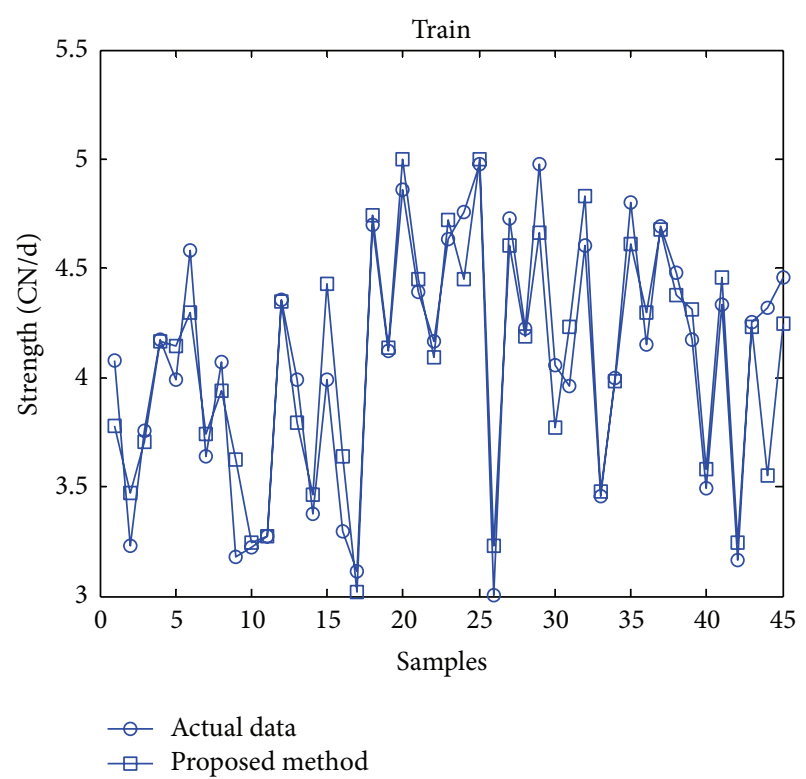

(a) Strength

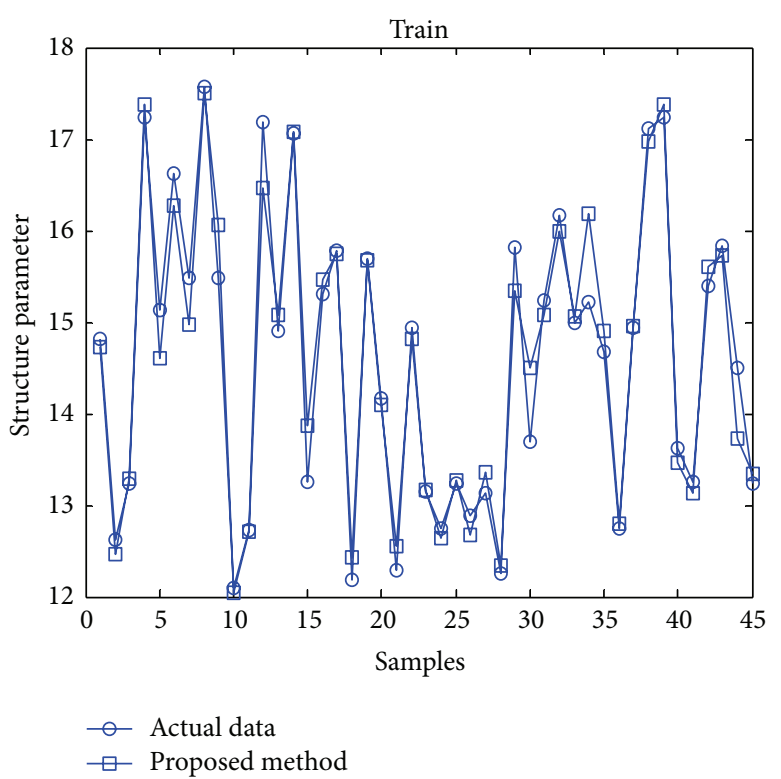

(b) Structure parameter

FIGURE 4: Training results of the proposed GA-IPSO-RNN model.

constrains, and function approximation [12-15]. Because of its robustness and fast training rate, RNN becomes the most suitable choice for real-time signal processing of an unknown nonlinear dynamical system. Qiao and Han [16] proposed a self-organizing RNN to model and identify nonlinear dynamical systems. Wang and Liu [17] presented a self-correcting RNN-based forecasting method for adapting changing conditions.
As we all known, every single algorithm has its own shortcomings which are difficult to overcome by improving itself. In that case, combining several artificial intelligence (AI) approaches is a new study trend in recent years. They can fully promote their respective superiorities while avoid their respective defects to enhance their optimizing effects. In the literatures, particle swarm optimization (PSO) is a popular algorithm because of its easy implementation 


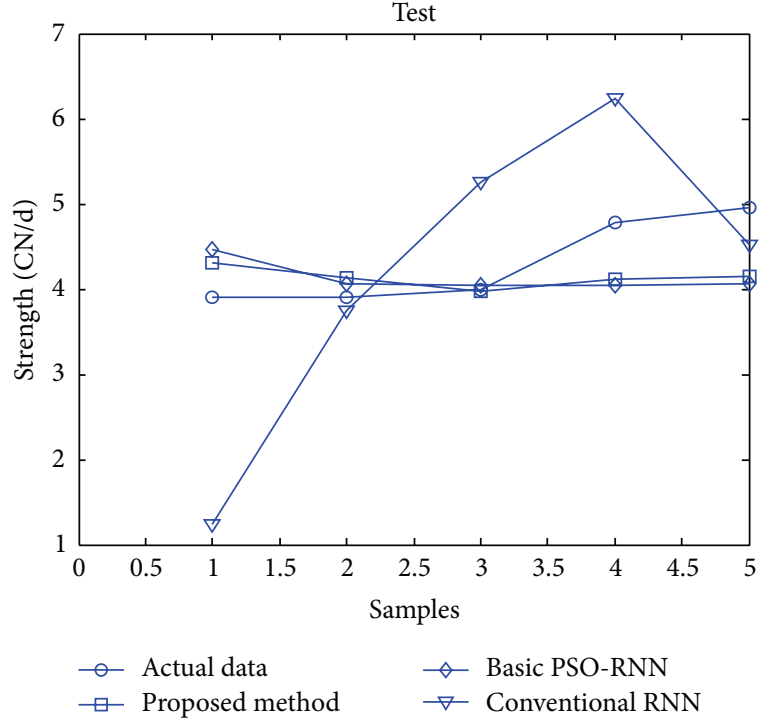

(a) Strength

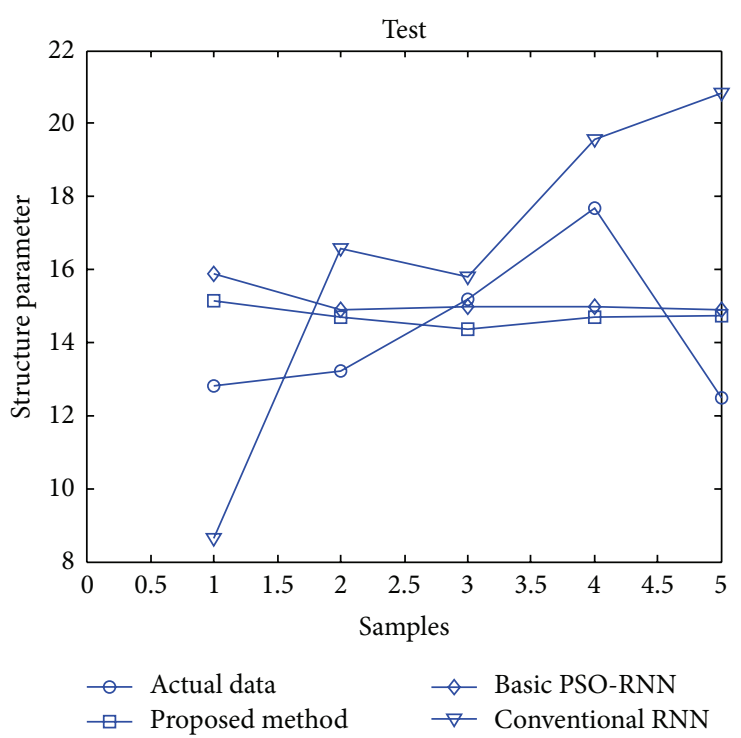

(b) Structure parameter

FIGURE 5: Performance of the proposed method, basic PSO-RNN, and conventional RNN.

procedure and high performance. Hu et al. [18] proposed an immune cooperative PSO algorithm for fault-tolerant routing optimization in heterogeneous wireless sensor networks. Alfi [19] introduced an adaptive mutation mechanism and a dynamic inertia weight to the PSO to estimate the unknown parameters in nonlinear systems. Behrang et al. [20] used the PSO to predict monthly average daily global solar radiation on horizontal surface for different regions of Iran. Mahor and Rangnekar [21] presented a PSO with self-adaptive inertia weight to determine the optimal short-term generation schedule of real operated cascaded hydroelectric system located at Narmada river in Madhya Pradesh, India. Tang et al. [22] proposed a novel PSO combined with a modified simulated binary crossover (MSBX). Luitel and Venayagamoorthy [23] combined quantum principles to the PSO and neural networks to optimize multiple-input-multiple-output learning systems. Vasumathi and Moorthi [24] added the PSO to adaptive neural network for harmonic isolation and reduction. Oh et al. [25] designed a polynomial RNN which optimized by the PSO for handling high-dimensional pattern classification problems. Huang and Wang [26] combined orthogonal least squares and enhanced PSO to RNN for realtime power dispatch of practical Taiwan Power Company systems. Li and Liu [27] proposed a hybrid algorithm with modified PSO, simulated annealing, and RNN to predict the product specifications melt index.

In this paper, we propose a neural network model with a GA-IPSO hybrid algorithm for bidirectional optimization of PAN-based carbon fiber. We use the nearest neighborclustering algorithm (NNCA) to decide the hidden layer nodes number of neural network, the GA-IPSO hybrid algorithm is used to tune the parameters in the network. By this model, for one direction, we predict the carbon fiber properties; for the other direction, we obtain produce scheme for new type carbon fiber.
The main contributions of this paper are as follows: (1) we propose a novel GA-IPSO hybrid algorithm to neural network, which pushes forward the development of AI; (2) by the proposed model, we can online monitor and control manufacture process of carbon fiber, forecast the product properties in real time, adjust produce parameters in time; (3) also, we can give out a reference produce scheme for new type carbon fiber which meets expect product properties, guides practical manufacture process, and prevents wasting time and money for putting into production directly.

The rest of this paper is organized as follows. In Section 2, the meaning and means of bidirectional optimization of PAN-based carbon fiber production is briefly reviewed. Section 3 describes the proposed optimization algorithm. In Section 4, practical test results of the proposed method by employing actual production and experiment data are illustrated. Comparisons with the other methods are also included. Conclusions are given in Section 5.

\section{The Carbon Fiber Production and Its Bidirectional Optimization}

As described in introduction, the production process of PANbased carbon fiber is a typical unknown nonlinear dynamical complex system. It is a key investigation field to keep the properties of carbon fiber stable and improve the properties as possible as we can. For this reason, we should monitor the production process of carbon fiber, observe producing parameters in real-time, accurately predict product properties online, then we can find out defects of carbon fiber ahead of time, adjust produce parameters in time, avoid generating carbon fiber of poor quality, and prevent wasting of production materials.

Designing new types carbon fiber with superior properties is another urgent task for engineers and scholars. 
TABLE 1: Experiment data of carbon fiber production process.

\begin{tabular}{|c|c|c|c|c|c|c|c|c|}
\hline No. & $\begin{array}{c}\text { Viscosity average } \\
\text { molecular weight }\left(10^{4}\right)\end{array}$ & $\begin{array}{c}\text { Conversion } \\
\text { ratio }(\%)\end{array}$ & $\begin{array}{c}\text { Solid } \\
\text { content }(\%)\end{array}$ & $\begin{array}{c}\text { Spinning jet } \\
\text { drawing ratio (\%) }\end{array}$ & $\begin{array}{l}\text { Coagulating bath } \\
\text { temperature }\left({ }^{\circ} \mathrm{C}\right)\end{array}$ & $\begin{array}{c}\text { Total } \\
\text { drawing ratio }\end{array}$ & $\begin{array}{l}\text { Strength } \\
(\mathrm{CN} / \mathrm{d})\end{array}$ & $\begin{array}{l}\text { Structure } \\
\text { parameter }\end{array}$ \\
\hline 1 & 8.9 & 94.5 & 20.8 & -50.3 & 14 & 6.33 & 4.08 & 14.82 \\
\hline 2 & 6.3 & 91.0 & 20.0 & -59.7 & 15 & 5.89 & 3.23 & 12.63 \\
\hline 3 & 11.6 & 92.0 & 20.4 & -50.5 & 14 & 6.03 & 3.76 & 13.24 \\
\hline 4 & 8.8 & 94.8 & 21.8 & -63.4 & 13 & 6.65 & 4.17 & 17.24 \\
\hline 5 & 7.0 & 81.8 & 17.9 & -63.4 & 15 & 6.32 & 3.99 & 15.14 \\
\hline 6 & 8.2 & 85.5 & 21.7 & -59.5 & 15 & 5.49 & 4.58 & 16.61 \\
\hline 7 & 7.2 & 89.8 & 19.5 & -53.1 & 13 & 5.88 & 3.64 & 15.49 \\
\hline 8 & 8.9 & 82.5 & 17.5 & -56.8 & 19 & 6.38 & 4.07 & 17.57 \\
\hline 9 & 8.0 & 83.4 & 18.6 & -62.1 & 17 & 5.72 & 3.18 & 15.48 \\
\hline 10 & 11.7 & 90.6 & 17.9 & -53.8 & 16 & 6.47 & 3.22 & 12.10 \\
\hline 11 & 11.5 & 82.8 & 18.7 & -64.8 & 17 & 5.79 & 3.27 & 12.73 \\
\hline 12 & 6.3 & 95.1 & 19.6 & -54.9 & 16 & 6.37 & 4.36 & 17.18 \\
\hline 13 & 10.4 & 98.6 & 20.2 & -68.3 & 17 & 6.41 & 3.99 & 14.91 \\
\hline 14 & 7.6 & 93.1 & 19.7 & -55.4 & 16 & 5.88 & 3.38 & 17.07 \\
\hline 15 & 8.5 & 84.6 & 22.3 & -65.3 & 18 & 5.04 & 3.99 & 13.26 \\
\hline 16 & 9.3 & 89.8 & 20.1 & -53.8 & 16 & 5.66 & 3.30 & 15.31 \\
\hline 17 & 11.7 & 79.4 & 22.7 & -55.8 & 19 & 5.85 & 3.11 & 15.78 \\
\hline 18 & 8.5 & 96.9 & 20.8 & -51.8 & 14 & 5.54 & 4.70 & 12.19 \\
\hline 19 & 11.9 & 96.4 & 22.7 & -61.5 & 13 & 5.39 & 4.12 & 15.69 \\
\hline 20 & 7.8 & 95.0 & 18.4 & -63.7 & 13 & 6.64 & 4.86 & 14.17 \\
\hline 21 & 10.2 & 82.7 & 21.1 & -60.9 & 13 & 5.86 & 4.39 & 12.30 \\
\hline 22 & 10.0 & 90.1 & 18.7 & -58.5 & 15 & 6.78 & 4.17 & 14.94 \\
\hline 23 & 9.2 & 77.5 & 21.0 & -62.9 & 16 & 5.78 & 4.63 & 13.16 \\
\hline 24 & 10.2 & 86.4 & 21.2 & -63.0 & 15 & 6.54 & 4.76 & 12.74 \\
\hline 25 & 10.0 & 83.9 & 17.4 & -63.6 & 18 & 5.79 & 4.98 & 13.23 \\
\hline 26 & 7.1 & 80.6 & 18.5 & -62.7 & 17 & 6.62 & 3.00 & 12.88 \\
\hline 27 & 6.8 & 80.9 & 18.3 & -68.9 & 18 & 6.51 & 4.73 & 13.13 \\
\hline 28 & 12.0 & 86.3 & 21.0 & -54.2 & 19 & 5.75 & 4.23 & 12.26 \\
\hline 29 & 7.0 & 79.1 & 22.1 & -64.2 & 19 & 5.43 & 4.98 & 15.81 \\
\hline 30 & 6.2 & 90.2 & 19.1 & -54.7 & 14 & 6.58 & 4.06 & 13.69 \\
\hline 31 & 9.4 & 87.4 & 21.7 & -52.4 & 13 & 6.90 & 3.96 & 15.23 \\
\hline 32 & 11.3 & 92.3 & 21.1 & -62.1 & 17 & 5.66 & 4.60 & 16.17 \\
\hline 33 & 10.0 & 92.4 & 17.0 & -59.0 & 13 & 6.34 & 3.46 & 14.99 \\
\hline 34 & 7.1 & 91.0 & 20.6 & -59.2 & 16 & 5.88 & 4.00 & 15.21 \\
\hline 35 & 8.2 & 77.7 & 19.3 & -63.2 & 16 & 6.67 & 4.80 & 14.67 \\
\hline 36 & 8.8 & 78.5 & 22.5 & -65.4 & 19 & 6.54 & 4.15 & 12.74 \\
\hline 37 & 11.9 & 84.0 & 17.0 & -57.0 & 16 & 5.33 & 4.69 & 14.94 \\
\hline 38 & 6.9 & 88.7 & 19.8 & -63.2 & 15 & 6.72 & 4.48 & 17.12 \\
\hline 39 & 11.1 & 91.4 & 19.5 & -58.3 & 17 & 6.98 & 4.17 & 17.24 \\
\hline 40 & 9.9 & 86.0 & 19.8 & -66.8 & 18 & 6.03 & 3.49 & 13.62 \\
\hline 41 & 8.3 & 95.0 & 21.6 & -66.7 & 16 & 6.77 & 4.33 & 13.25 \\
\hline 42 & 7.1 & 92.8 & 18.9 & -55.1 & 15 & 6.18 & 3.17 & 15.39 \\
\hline 43 & 8.6 & 98.3 & 21.7 & -62.3 & 14 & 5.31 & 4.25 & 15.84 \\
\hline 44 & 8.9 & 88.7 & 19.8 & -61.6 & 17 & 5.40 & 4.32 & 14.50 \\
\hline
\end{tabular}


TABle 1: Continued.

\begin{tabular}{ccccccccc}
\hline No. & $\begin{array}{c}\text { Viscosity average } \\
\text { molecular weight }\left(10^{4}\right)\end{array}$ & $\begin{array}{c}\text { Conversion } \\
\text { ratio (\%) }\end{array}$ & $\begin{array}{c}\text { Solid } \\
\text { content }(\%)\end{array}$ & $\begin{array}{c}\text { Spinning jet } \\
\text { drawing ratio (\%) }\end{array}$ & $\begin{array}{c}\text { Coagulating bath } \\
\text { temperature }\left({ }^{\circ} \mathrm{C}\right)\end{array}$ & $\begin{array}{c}\text { Total } \\
\text { drawing ratio }\end{array}$ & $\begin{array}{c}\text { Strength } \\
(\mathrm{CN} / \mathrm{d})\end{array}$ & $\begin{array}{c}\text { Structure } \\
\text { parameter }\end{array}$ \\
\hline 45 & 6.7 & 84.2 & 17.2 & -60.8 & 14 & 5.81 & 4.46 & 13.24 \\
46 & 11.0 & 98.0 & 22.7 & -74.6 & 12 & 6.89 & 3.90 & 12.82 \\
47 & 9.5 & 92.2 & 20.3 & -50.5 & 17 & 5.92 & 3.91 & 13.20 \\
48 & 7.7 & 78.2 & 17.2 & -63.4 & 19 & 6.17 & 3.99 & 15.19 \\
49 & 9.5 & 79.3 & 18.1 & -67.4 & 13 & 6.50 & 4.78 & 17.69 \\
50 & 7.4 & 90.4 & 21.3 & -55.3 & 18 & 6.65 & 4.96 & 12.49 \\
\hline
\end{tabular}

In the past, we always make a production scheme of new type carbon fiber through former experiences, then we produce this kind of carbon fiber according to the proposed experiential scheme by testing production line. After obtaining carbon fiber from the practical experiment, we put the carbon fiber to physical and chemical instruments to test its property indices, compare these indices to expect value, get the disparity, adjust several produce parameters in proposed scheme, produce carbon fiber according to adjusted scheme by testing production line again, repeat executing produce, test, and adjust process until we gain the carbon fiber which is satisfactory to us. This method wastes time, energy, and cost. The most terrible thing is that, if we do not choose the appropriate parameters of control devices, it will result in oscillation and unstability of the whole system, which brings in severe loss. Therefore, it is a better choice to carry out computer simulation, analyze and synthesize all the antecedent produce schemes, avoid one-sided decision made by workers from their personal experience and interests, and give out a more scientific and proper control parameters from an optimal production scheme.

In this paper, we propose a bidirectional optimization model of carbon fiber production process based on RNN. We adopt the nearest neighbor-clustering algorithm to select a suitable set of centers of the network and introduce a GA-IPSO hybrid algorithm to tune the parameters of the network. When we take production parameters as input and properties indices as output, this model can monitor production parameters in real time and predict properties indices online. When we take properties indices as input and production parameters as output, this model can be viewed as a designer of developing new type carbon fiber. All these bidirectional optimization functions are as shown in Figure 1.

\section{The GA-IPSO Hybrid Algorithm for Neural Network}

3.1. RBF Neural Network. The RNN is a typical supervised learning feed-forward neural network which is put forward by Moody and Darken in 1989 [28]. It is a three-layer network composed of input layer, hidden layer, and output layer. The input layer just receives and transforms input signal to hidden layer; the hidden layer adapts radial basis functions as activation function; the output layer always gives the final responses through simple linear functions.
The common radial basis functions are listed as follows:

$$
\begin{gathered}
f(x)=\exp ^{-(x / \sigma)^{2}}, \\
f(x)=\frac{1}{\left(\sigma^{2}+x^{2}\right)^{\alpha}}, \quad \alpha>0, \\
f(x)=\left(a^{2}+x^{2}\right)^{\beta}, \quad \alpha<\beta<1 .
\end{gathered}
$$

As Gaussian function is the outstanding representative among them, we use it as activation function of hidden layer in this paper:

$$
u_{j}=G\left(\left\|x_{d}-c_{j}\right\|\right)=\exp \left(-\frac{\left\|x_{d}-c_{j}\right\|^{2}}{2 \sigma_{j}^{2}}\right), \quad j=1,2, \ldots, N_{h},
$$

where $u_{j}$ is the output of node $j$ in hidden layer, $x_{d}=$ $\left(x_{1}, x_{2}, \ldots, x_{N_{i}}\right)^{T}$ is the input vector, $N_{i}$ is the number of input nodes, $c_{j}$ is the $j$ th center node in the hidden layer, $\sigma_{j}$ is the width of the $j$ th center node, $N_{h}$ is the number of hidden nodes, and $\|\cdot\|$ denotes Euclidean distance.

The output is formed by a linear combination of the hidden layer responses

$$
y_{i}=\sum_{j=1}^{N_{h}} \omega_{i j} u_{j}-\theta=W_{i}^{T} U, \quad i=1,2, \ldots, N_{o},
$$

where $W_{i}=\left(\omega_{i 1}, \omega_{i 2}, \ldots, \omega_{i N_{h}}-\theta\right)^{T}$ represents the linking weight of the node in hidden layer and the node in output layer, $U=\left(u_{1}, u_{2}, \ldots, u_{N_{h}}, 1\right)^{T}, N_{o}$ is the number of output nodes.

First of all, we should confirm the structure of RNN, the number of input nodes $N_{i}$, and the number of output nodes $N_{o}$ are decided by production parameters and product properties indices, respectively, according to their optimization directions. The crux is fixing the number of hidden nodes $N_{h} . N_{h}$ influences the capability of network directly. If the network structure is redundant, it will occupy mass software and hardware while training and testing, even leads to the network over fit, obtains excellent performance when it is training while shows horrible results during testing. If the network structure is too small, the network cannot stand for global information. At present, there is not a uniform method 


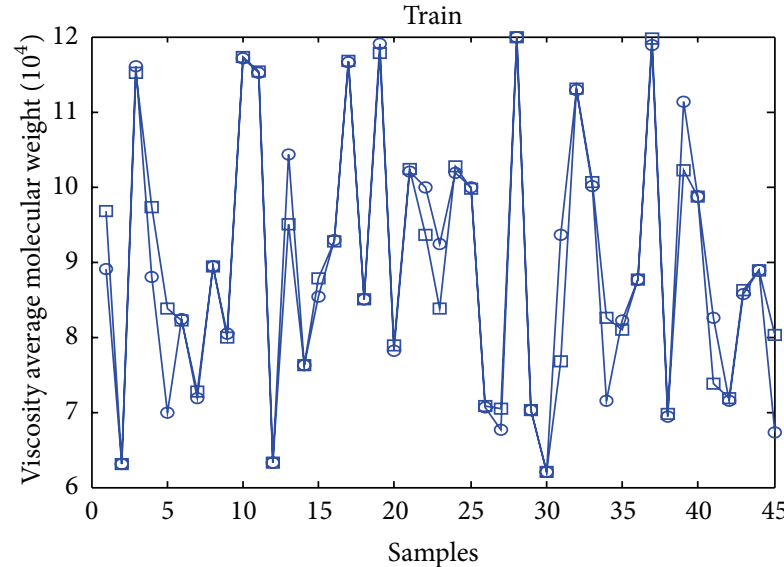

$\multimap$ Actual data

$\square$ Proposed method

(a) Viscosity average molecular weight

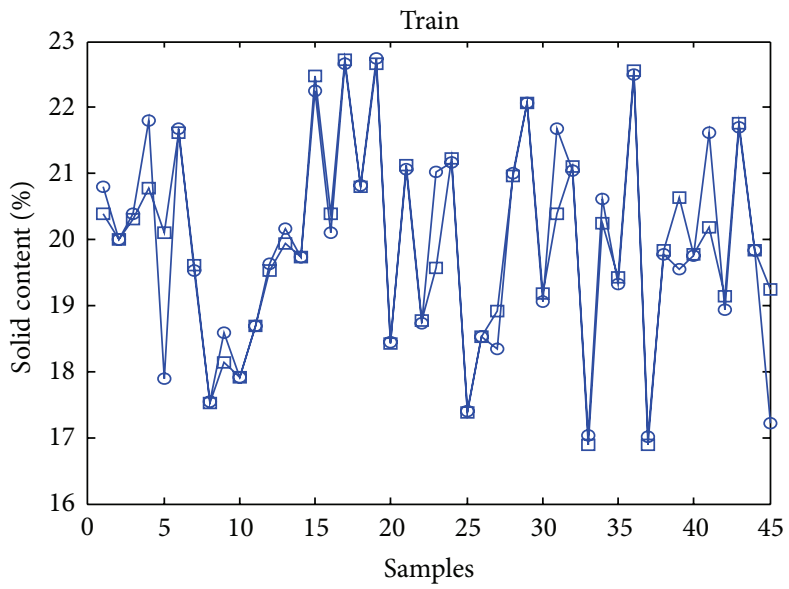

- - Actual data

$\neg$ Proposed method

(c) Solid content

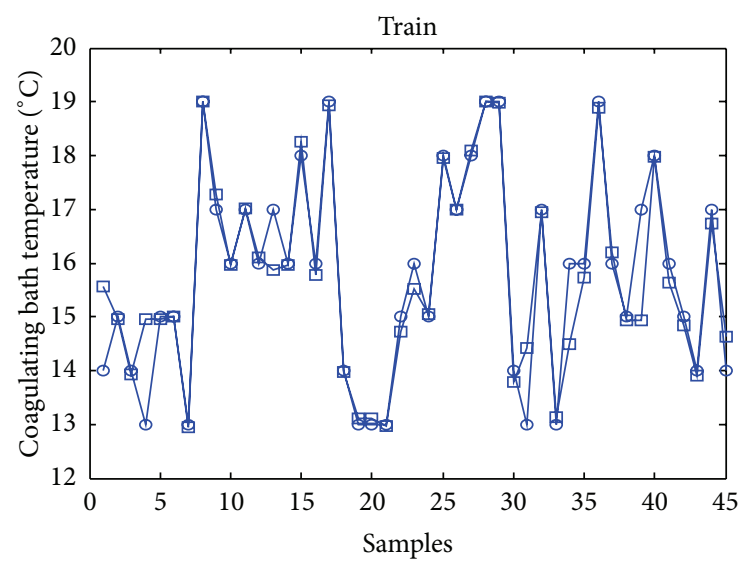

- Actual data

$\neg$ Proposed method

(e) Coagulating temperature

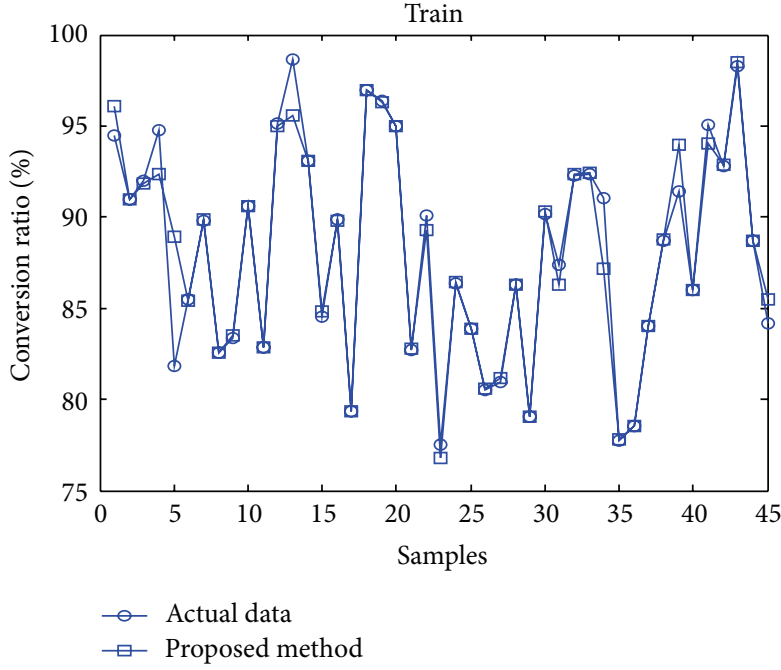

(b) Conversion ratio

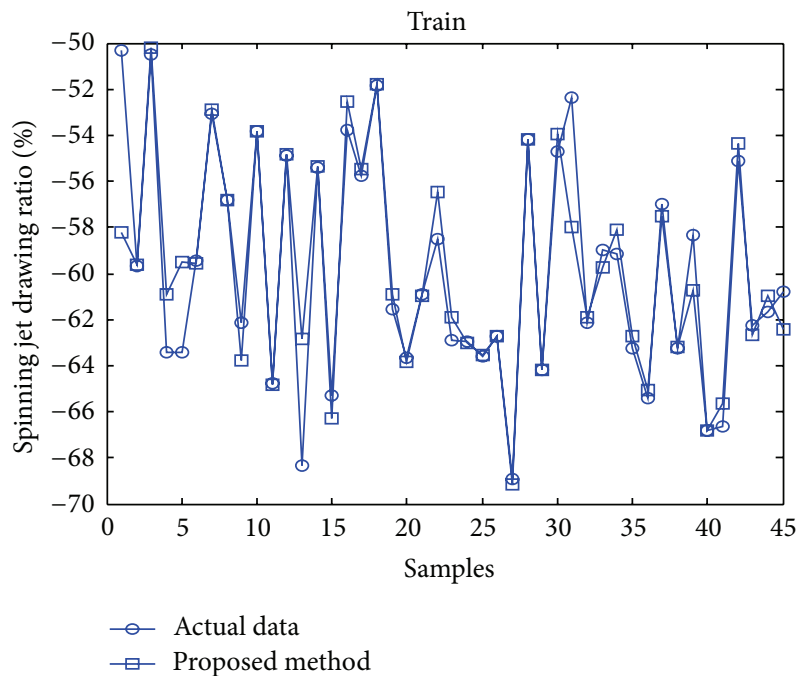

(d) Spinning jet drawing ratio

Train

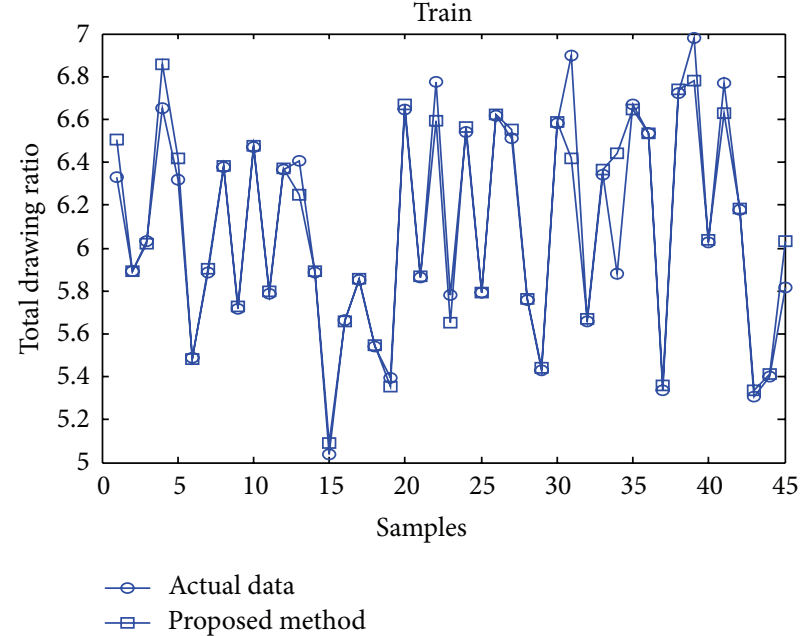

(f) Total drawing ratio

FIGURE 6: Training results of the proposed GA-IPSO-RNN model. 
to determine $N_{h}$. Generally, $N_{h}$ is selected by trail and error referring to experience expression given by

$$
N_{h}=\sqrt{N_{i}+N_{o}}+\xi, \quad \xi=1,2, \ldots, 10
$$

In this work, we adopt the nearest neighbor-clustering algorithm to acquire $N_{h}$. The NNCA is firstly presented in 1973 [29]. This algorithm is an online self-adaption cluster algorithm, and it does not need to fix $N_{h}$ first. It collects data, constructs structure, and trains network at the same time, which is called online learning. It displays as a gradual increase method, which starts from a simple network and gradually increases the number of hidden nodes until it fulfills the purpose. While clustering is finished, the optimal network is formed. The nearest neighbor-clustering algorithm can be shown in Figure 2.

The specific procedures of the nearest neighborclustering algorithm are shown below.

Step 1. Choose a proper width $r$ of Gaussian function, here it means $\sigma$, define a vector $A(l)$ to store the sum of output vectors of each class, and also define a counter $B(l)$ to calculate the numbers of samples belong to each class, in which $l$ means how many classes there exist.

Step 2. Begin from the first sample $\left(x_{1 d}, y_{1}\right)$, set up $x_{1 d}$ as the first cluster center, sign it as follows: $c_{1}=x_{1 d}, A(1)=$ $y_{1}, B(1)=1$. At this time, an original RNN is formed. So far, this RNN includes only one node in hidden layer, $c_{1}$ is the center of this node, the weight vector from this node to output layer is $w_{1}=A(1) / B(1)$.

Step 3. Consider the second sample $\left(x_{2 d}, y_{2}\right)$, calculate the distance between $x_{2 d}$ and $c_{1}$, and mark it as $\left\|x_{2 d}-c_{1}\right\|$. If $\left\|x_{2 d}-c_{1}\right\| \leq r, c_{1}$ pertains to the nearest neighbor-cluster of $x_{2 d}$, then $A(1)=y_{1}+y_{2}, B(1)=2, w_{1}=A(1) / B(1)$. If $\left\|x_{2 d}-c_{1}\right\|>r, x_{2 d}$ can be viewed as another cluster center, then $c_{2}=x_{2 d}, A(2)=y_{2}, B(2)=1$. Add a new node to hidden layer of existing RNN, the weight vector from this node to output layer is $w_{2}=A(2) / B(2)$.

Step 4. Think about the samples one by one. Assuming that the nodes number of hidden layer is $M$ when we take the $k$ th sample $\left(x_{k d}, y_{k}\right)(k=3,4, \ldots, N)$ into account, which means the number of cluster centers is $M$, the centers are $c_{1}, c_{2}, \ldots, c_{M}$, respectively. At this situation, calculate distances between $x_{k d}$ and these cluster centers which described as $\left\|x_{k d}-c_{i}\right\|, i=1,2, \ldots, M$. Set up $\left\|x_{k d}-c_{j}\right\|$ as the minimal distance, in this way, $c_{j}$ is the nearest neighbor cluster of $x_{k d}$. If $\left\|x_{k d}-c_{j}\right\| \leq r, c_{j}$ pertains to the nearest neighbor-cluster of $x_{k d}$, then $A(j)=A(j)+y_{k}, B(j)=B(j)+1$. When $i \neq j, i=$ $1,2, \ldots, M$, keep $A(i)$ and $B(i)$ as the same as before, weight vectors from hidden layer to output layer are $w_{i}=A(i) / B(i)$, $i=1,2, \ldots, M$. If $\left\|x_{k d}-c_{j}\right\|>r, x_{k d}$ becomes a new cluster center, then $c_{M+1}=x_{k d}, M=M+1, A(M)=y_{k}, B(M)=1$, $A(i)$ and $B(i)$ will not change $(i=1,2, \ldots, M-1)$, add the $M$ th node to hidden layer of RNN which is built in last step, the weight vector from it to output layer is $w_{M}=A(M) / B(M)$.
Step 5. Do Step 4 again and again until the last sample has been regarded, finish it.

After executing the nearest neighbor-clustering algorithm, we obtain the number of centers and the parameters of RNN which means the position and width of centers, weights from hidden nodes to output nodes at the same time. However, in this paper, we just use the nearest neighborclustering algorithm to determine the number of hidden nodes, which is the number of centers. We will tune the position and width of centers, weights from hidden nodes to output nodes by proposed GA-IPSO which is described in Section 3.2. But the parameters of RNN we gained from the nearest neighbor-clustering algorithm will be taken as initial particle swarm range.

\subsection{GA-IPSO Hybrid Algorithm}

3.2.1. Basic PSO Algorithm. PSO algorithm is an efficient population-based stochastic optimization technique inspired by social behavior of bird flocks or fish schools which is originally developed by Kennedy and Eberhart in 1995 [30]. Since PSO requires short computer time and less memory, it has been successfully applied to a wide range of optimization applications.

The principle of PSO: for a $D$-dimension problem, it maintains a population of particles distribute uniformly around a $D$-dimension search space at first. Suppose the size of the swarm is $m$, the population is represented as $x=$ $\left(x_{1}, x_{2}, \ldots, x_{m}\right)^{T}$. Each particle in this population indicates a potential solution to the problem. A particle is recorded from two aspects of its position and velocity: the position is represented as $x_{i d}=\left(x_{i 1}, x_{i 2}, \ldots, x_{i D}\right)$, and the velocity is represented as $v_{i d}=\left(v_{i 1}, v_{i 2}, \ldots, v_{i D}\right)$. The position is associated with the fitness. The best fitness the particle has achieved is considered as its current best position, marked it as $p_{i d}=\left(p_{i 1}, p_{i 2}, \ldots, p_{i D}\right)$. The best position in the whole population is recorded as $p_{g d}=\left(p_{g 1}, p_{g 2}, \ldots, p_{g D}\right)$. In every generation, each particle updates its status in light of three criterion: (1) its own inertia; (2) the cognitive information $p_{i d}$; (3) the social information $p_{g d}$. These particles in the population tend to cluster together by updating their status until the optimum solution is obtained at last.

For the $t$ iteration, the velocity and position of each particle are updated in the following manner:

$$
\begin{gathered}
v_{i d}^{t+1}=w v_{i d}^{t}+c_{1} r_{1}\left(p_{i d}^{t}-x_{i d}^{t}\right)+c_{2} r_{2}\left(p_{g d}^{t}-x_{i d}^{t}\right) \\
x_{i d}^{t+1}=x_{i d}^{t}+v_{i d}^{t+1},
\end{gathered}
$$

where $w$ is the inertia weight, which decides influence on the next generation of the particle current velocity. $c_{1}$ and $c_{2}$ are acceleration constants, called the cognitive and social parameter, respectively. $r_{1}$ and $r_{2}$ are uniform random numbers between 0 and 1 . 


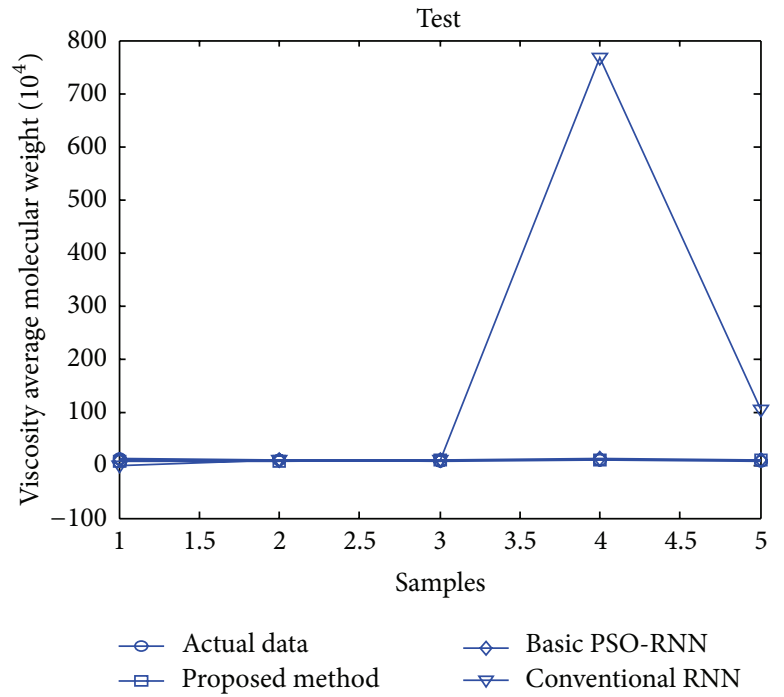

(a) Viscosity average molecular weight

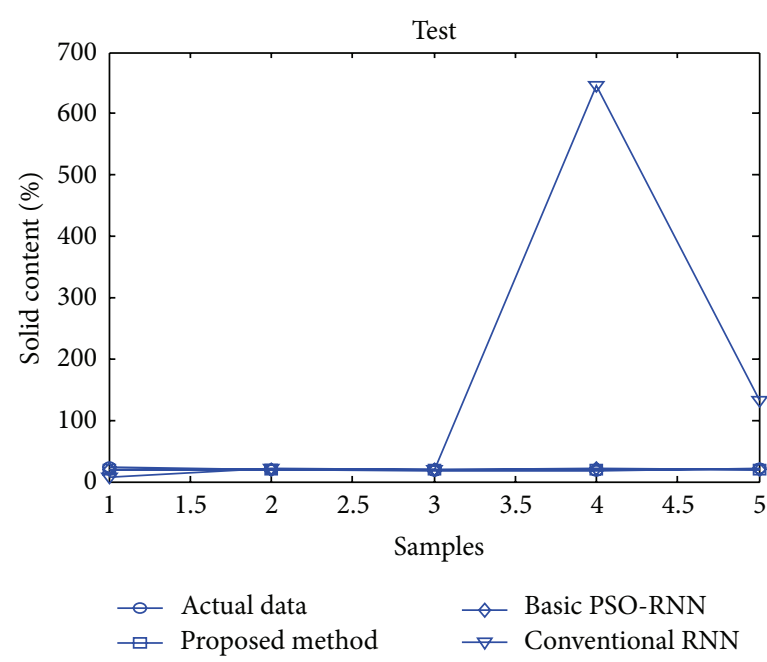

(c) Solid content

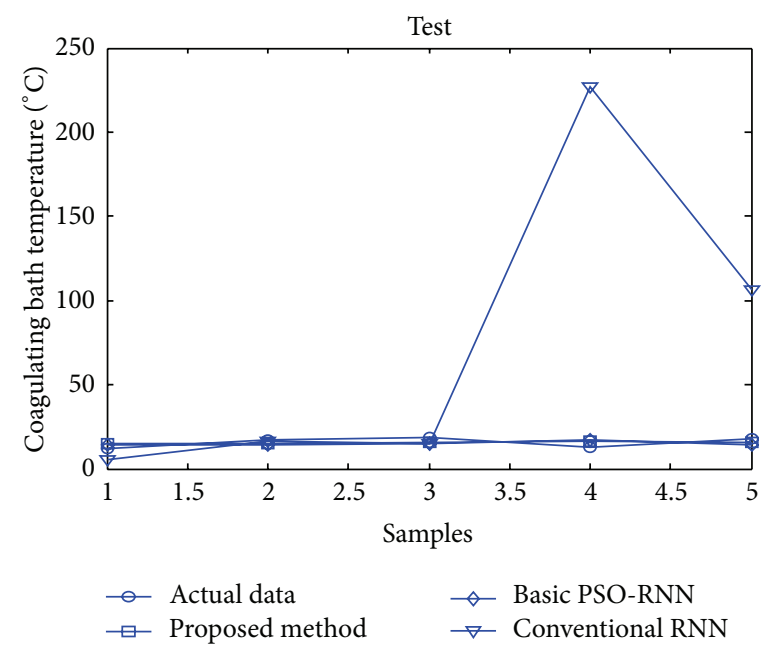

(e) Coagulating temperature

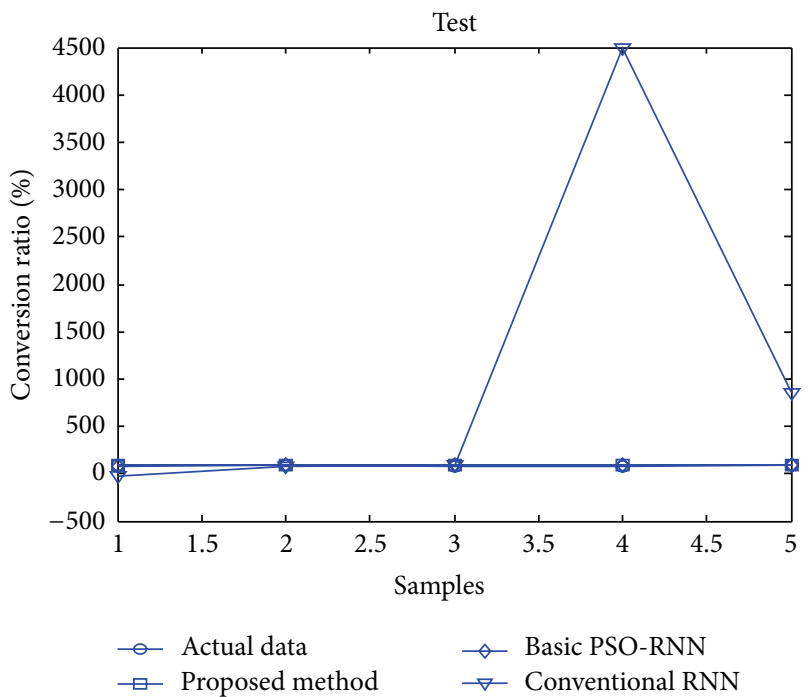

(b) Conversion ratio

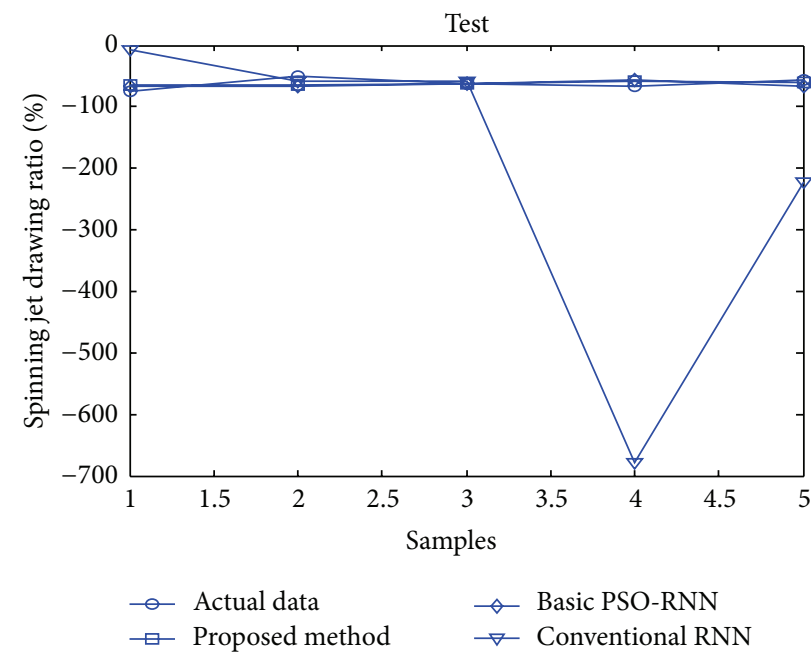

(d) Spinning jet drawing ratio

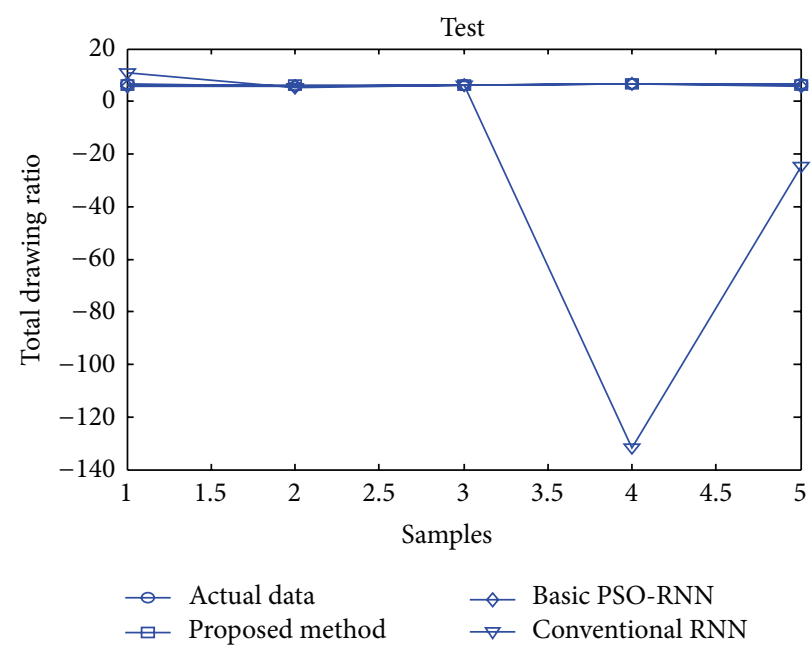

(f) Total drawing ratio

FIGURE 7: Performance of the proposed method, basic PSO-RNN, and conventional RNN. 
Commonly, $c_{1}=c_{2}=2.05, w_{\max }=0.9, w_{\min }=0.4$, for $T$ is the maximal number of iterations, $w$ of every iteration is calculated according to:

$$
w=w_{\max }-\frac{t}{T}\left(w_{\max }-w_{\min }\right)
$$

3.2.2. GA-IPSO Hybrid Algorithm. Furthermore, although PSO has good convergent property, if the initial population of particles cannot effectively cover the whole region, the population diversity will be greatly reduced after some iterations, as such the particles will converge easily to a local optimum. Aim to avoid the limitations and enhance the superiorities, due to the fact that PSO utilizes $w, p_{i d}$ and $p_{g d}$, to modify the current search point to prevent the particles flying in the same direction; in this paper, we employ dynamic-adjust $w$ and introduce a penalty factor $\left(\theta_{1}, \theta_{2}\right)$ for coordinating the guides of $p_{i d}$, and $p_{g d}$. In that case, the formulas (7) can be rewritten as

$$
v_{i d}^{t+1}=w^{t} v_{i d}^{t}+\theta_{1}^{t} c_{1} r_{1}\left(p_{i d}^{t}-x_{i d}^{t}\right)+\theta_{2}^{t} c_{2} r_{2}\left(p_{g d}^{t}-x_{i d}^{t}\right) .
$$

(1) Particle and Its Fitness. The parameters of RNN needed to be tuned are positions and widths of hidden centers, weights between hidden layer and output layer. A group of parameters is a particle. Since numbers of input, hidden, output layer are $N_{i}, N_{h}, N_{o}$, the dimension of a particle is given by

$$
D=\left(N_{i}+1+N_{o}\right) \times N_{h} .
$$

Mark the output of RNN as $y$, the expect output as $\hat{y}$. The goal is to minimize the error between $y$ and $\hat{y}$. So the fitness function of particles is given by

$$
f=\frac{1}{2 n} \sum_{i=1}^{n}\left|y_{i}-\widehat{y}_{i}\right|^{2} .
$$

(2) Dynamic-Adjust Inertia Weight $w$. Inertia weight $w$ expresses the search length of the PSO. When $w$ is a large value, it is good for global optimum search owing to the fact that it can easily get out of local optimum; when $w$ is a small value, it is good for local optimum search and accelerate convergence. Although $w$ in formula (9) is not a constant, it cannot be called dynamic-adjust, it is just a kind of linear decrease. The linear decrease method has weak inspire ability of search direction though it is convenient to realize. Here, we dynamically adjust $w$ based on the change of the smoothness [31] of the object function. The definition of smoothness is shown below:

$$
a^{t}=\frac{1}{m} \sum_{i=1}^{m}\left|f\left(x_{i d}^{t}\right)-f\left(x_{\min d}^{t}\right)\right|,
$$

where $f\left(x_{i d}^{t}\right)$ is the fitness value of the $i$ th particle on iteration $t, f\left(x_{\min d}^{t}\right)$ is the fitness value of the best particle on iteration $t$, and these are expressed below:

$$
\begin{gathered}
f\left(x_{i d}^{t}\right)=f\left(x_{i 1}^{t}, x_{i 2}^{t}, \ldots, x_{i D}^{t}\right), \\
f\left(x_{\min d}^{t}\right)=\min _{i=1,2, \ldots, m} f\left(x_{i d}^{t}\right) .
\end{gathered}
$$

On every iteration, $w$ changes with $a^{t}$ like:

$$
\begin{gathered}
w^{t}=1.1^{-\lambda} w^{t-1}, \quad t=2, \ldots, T, \\
\lambda= \begin{cases}1, & a^{t}-a^{t-1}>0, \\
0, & a^{t}-a^{t-1}=0, \\
-1, & a^{t}-a^{t-1}<0 .\end{cases}
\end{gathered}
$$

When $a^{t}-a^{t-1}>0$, it indicates this iteration is emanative, the search step length should be decreased for precisely search in range around extremum; when $a^{t}-a^{t-1}<0$, it indicates this iteration is convergent, the search step length should be increased for accelerating search for extremum.

(3) Cognitive Parameter $c_{1}$ and Social Parameter $c_{2}$. In GAIPSO, we also change cognitive parameter $c_{1}$ and social parameter $c_{2}$ linearly as follows:

$$
\begin{aligned}
& c_{1}=c_{1 \min }+\frac{t}{T}\left(c_{1 \max }-c_{1 \min }\right), \\
& c_{2}=c_{2 \max }-\frac{t}{T}\left(c_{2 \max }-c_{2 \min }\right) .
\end{aligned}
$$

(4) Penalty Factor $\left(\theta_{1}, \theta_{2}\right)$. After calculating recent fitness of a particle on each iteration, we change the position of the particle a little, then calculate the fitness of the new position. If the new fitness is smaller than the resent fitness, it means the currents $p_{i d}$ and $p_{g d}$ cannot give out direction guidance precisely, then we compare the new fitness to the best fitness of this particle and the best fitness of the best particle in the population, respectively, to check out which one shows a bad answer. When the new fitness is smaller than the best fitness of this particle, it means the best position of this particle does not affect search direction correctly, then its influence should be decreased as a penalty, on the contrast, the cognitive influence should be increase. The mechanism is the same to the social influence expressed by the best position of the best particle in the population. The mathematical expression is showed below:

$$
\begin{gathered}
x_{\text {new } d}^{t}=x_{i d}^{t}-0.5 \operatorname{rand}(), \quad t=2, \ldots, T, \\
\theta_{1}^{t}=1.1^{-\alpha} \theta_{1}^{t-1},
\end{gathered}
$$



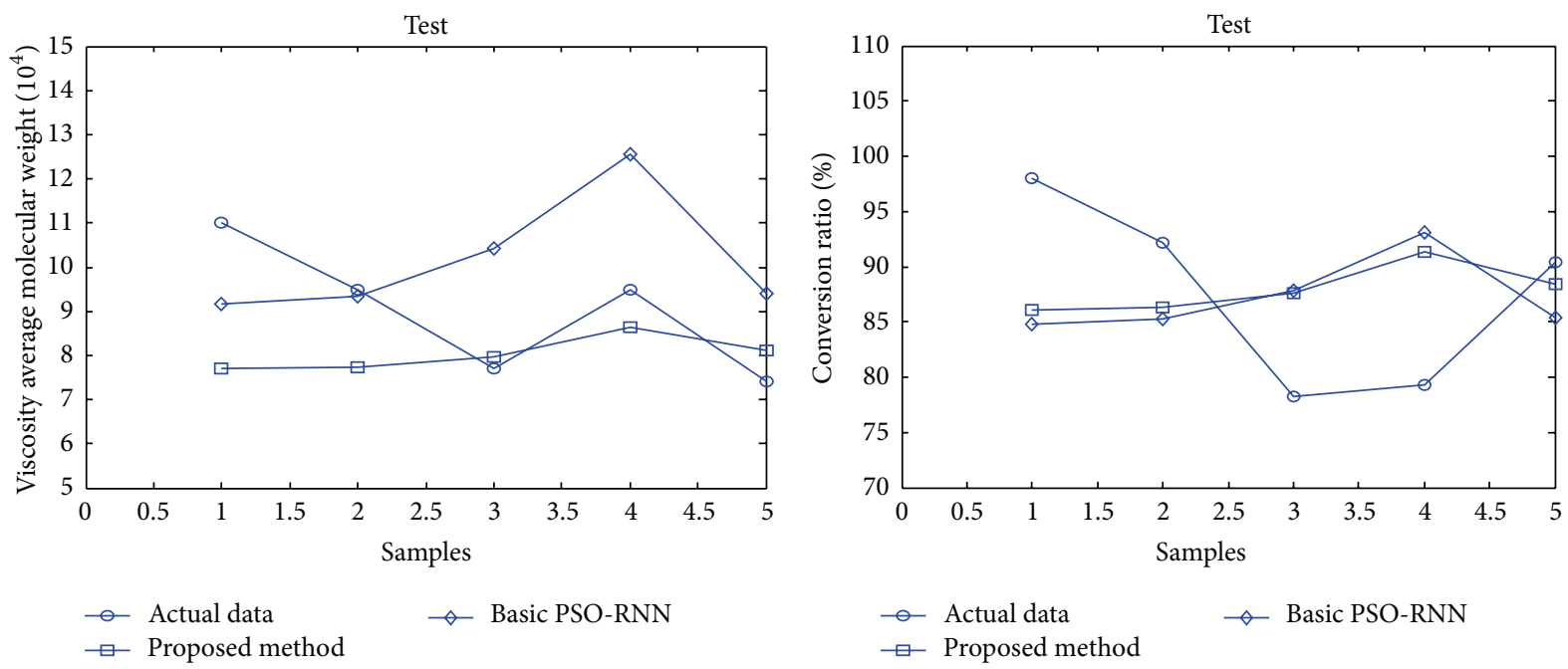

(b) Conversion ratio

(a) Viscosity average molecular weight

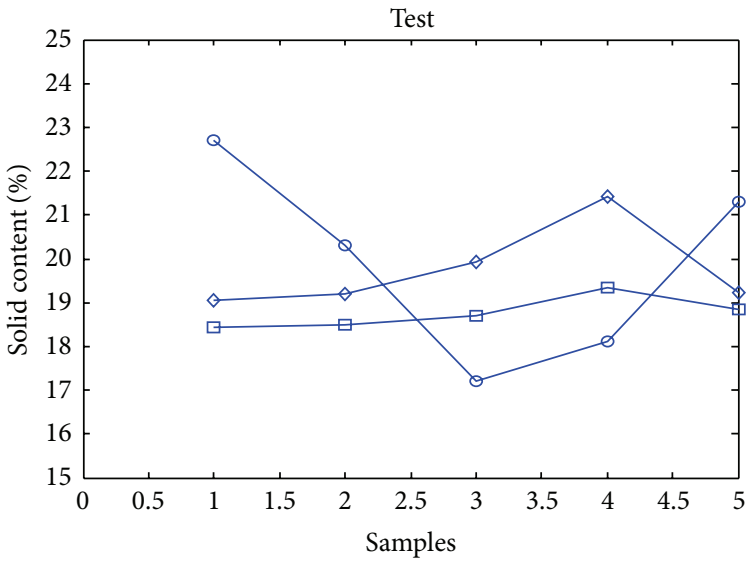

- Actual data $\square$ Proposed method

(c) Solid content

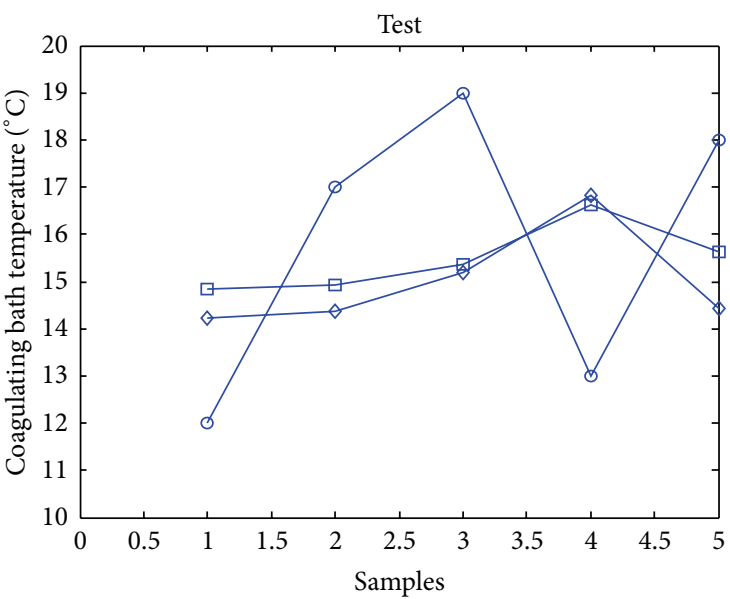

- - Actual data

—— Proposed method

(e) Coagulating temperature

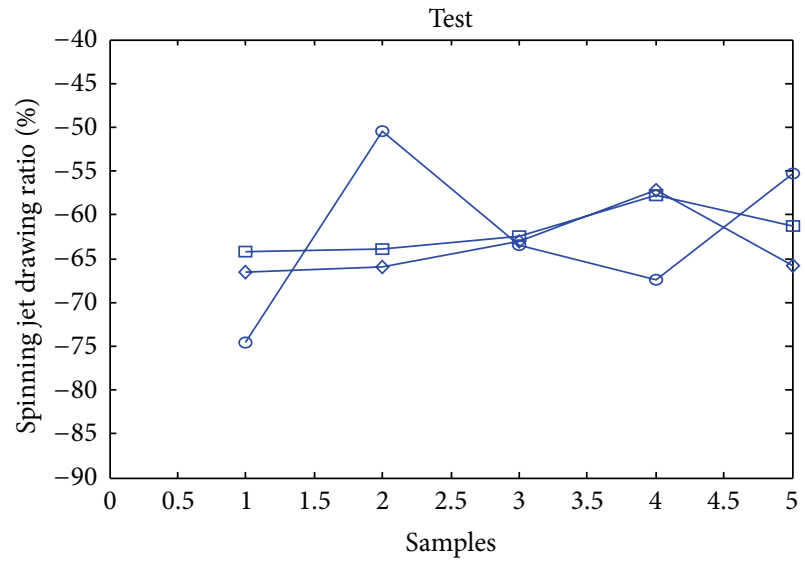

- - Actual data

$\neg$ Basic PSO-RNN

$\square$ Proposed method

(d) Spinning jet drawing ratio

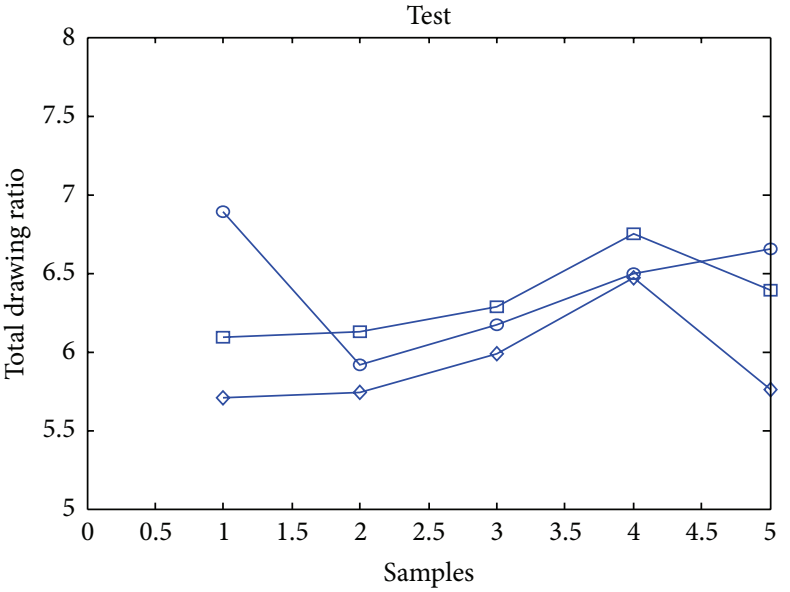

- - Actual data

$\neg$ Basic PSO-RNN

(f) Total drawing ratio

FIGURE 8: Performance comparison of the proposed method and basic PSO-RNN. 


$$
\begin{aligned}
& \alpha=\left\{\begin{array}{cc}
1, & \left(f\left(x_{\text {new } d}^{t}\right)-f\left(p_{i d}^{t}\right)<0\right) \\
& \cap\left(f\left(x_{\text {new } d}^{t}\right)-f\left(x_{i d}^{t}\right)<0\right), \\
0, & \left(f\left(x_{\text {new } d}^{t}\right)-f\left(p_{i d}^{t}\right)=0\right) \\
& \cap\left(f\left(x_{\text {new } d}^{t}\right)-f\left(x_{i d}^{t}\right)<0\right) \\
& \cup\left(f\left(x_{\text {new } d}^{t}\right)-f\left(x_{i d}^{t}\right) \geq 0\right), \\
-1, & \left(f\left(x_{\text {new } d}^{t}\right)-f\left(p_{i d}^{t}\right)>0\right) \\
& \cap\left(f\left(x_{\text {new } d}^{t}\right)-f\left(x_{i d}^{t}\right)<0\right),
\end{array}\right. \\
& \theta_{2}^{t}=1.1^{-\beta} \theta_{1}^{t-1} \\
& \beta=\left\{\begin{array}{cc}
1, & \left(f\left(x_{\text {new } d}^{t}\right)-f\left(p_{\text {gd }}^{t}\right)<0\right) \\
& \cap\left(f\left(x_{\text {new } d}^{t}\right)-f\left(x_{i d}^{t}\right)<0\right), \\
0, & \left(f\left(x_{\text {new } d}^{t}\right)-f\left(p_{\text {gd }}^{t}\right)=0\right) \\
& \cap\left(f\left(x_{\text {new } d}^{t}\right)-f\left(x_{i d}^{t}\right)<0\right) \\
& \cup\left(f\left(x_{\text {new } d}^{t}\right)-f\left(x_{i d}^{t}\right) \geq 0\right), \\
-1, & \left(f\left(x_{\text {new } d}^{t}\right)-f\left(p_{\text {gd }}^{t}\right)>0\right) \\
& \cap\left(f\left(x_{\text {new } d}^{t}\right)-f\left(x_{i d}^{t}\right)<0\right) .
\end{array}\right.
\end{aligned}
$$

(5) The GA-IPSO. With the iteration increasing, the population diversity will be reduced. Sometimes stagnation happens, it displays that $v$ is almost zero. For GA is famous for its cross and mutation operations, it can produce new particles, rebirth the whole population, and escape from the above terrible situation. So, we combine GA to the IPSO, and adopt $p_{i d}$ and $p_{g d}$ to execute cross and mutation operations, which means the new particles keeping the advantage of being guided by currents $p_{i d}$ and $p_{g d}$. The mechanism is showed below:

cross

$$
v_{i d c}^{t+1}=\eta p_{i d}^{t}+(1-\eta) p_{g d}^{t}=\left(v_{i 1 c}^{t+1}, v_{i 2 d}^{t+1}, \ldots, v_{i D}^{t+1}\right)
$$

mutation

$$
\begin{gathered}
d m=\lfloor 1+(D-1) \operatorname{rand}()\rfloor \\
v_{i d}^{t+1}=\left(v_{i 1 c}^{t+1}, \ldots, v_{i d m d}^{t+1} \operatorname{rand}(), \ldots, v_{i D}^{t+1}\right),
\end{gathered}
$$

where $\eta$ is a cross-coefficient between 0 and 1 , formula (20) is used to select which dimension will be mutation, \lfloor\rfloor indicates getting an integer downward.

The flowchart of GA-IPSO is indicated in Figure 3.

The specific procedures of the GA-IPSO are shown below.

Step 1. Set iteration as $t=1$. Initiate the parameters such as $w^{1}, \theta_{1}^{1}, \theta_{2}^{1}, c_{1 \min }, c_{1 \max }, c_{2 \min }, c_{2 \max }, \eta$, maximum $T$, swarm size $M$, error_goal.

Step 2. Initiate the particle swarm according to the range given by the nearest neighbor-clustering algorithm.

Step 3. Calculate the fitness of each particle in the population.

Step 4. Set up $p_{i d}$ and $p_{g d}$ of the first iteration.
Step 5. Set up $x_{i d}$ and $v_{i d}$ of each particle for next iteration.

Step 6. Update iteration $t=t+1$.

Step 7. Calculate the fitness of each particle in the new population.

Step 8. Update $p_{i d}$ and $p_{g d}$.

Step 9. Change the position of each particle in this population a little, calculate the new fitness, and update $\theta_{1}^{1}$ and $\theta_{2}^{1}$ according to formula (18).

Step 10. Update $x_{i d}$ and $v_{i d}$ of each particle according to (13)(17), (10), and (8).

Step 11. If $v=0$, execute GA, cross and mutation $v_{\mathrm{id}}$ according to formula (19)-(21).

Step 12. Do Step 6 Step 12 until fitness satisfies the error_goal or $t$ reaches $T$.

\section{Simulation and Results}

4.1. Data Preparation. Aiming to test and demonstrate the performance of the model we proposed, we used these data collected and organized from experiments in Table 1.

In these data, viscosity average molecular weight, conversion ratio, solid content, spinning jet drawing ratio, coagulating bath temperature and total drawing ratio are production parameters, strength, and structure parameters are property indices. These specific production parameters and property indices can relatively fully represent the whole manufacture process and product performance of PAN-based carbon fiber. In the bidirectional optimization process, we take the first 45 groups of samples as training dataset and the last 5 groups of samples as test dataset. We also make comparison between conventional RNN, basic PSO-RNN, and the proposed method, observe their performance in different ways which are defined as the following, respectively:

mean absolute error:

$$
\mathrm{MAE}=\frac{1}{n} \sum_{i=1}^{n}\left|y_{i}-\widehat{y}_{i}\right|,
$$

mean relative error:

$$
\operatorname{MRE}=\frac{1}{n} \sum_{i=1}^{n}\left|\frac{y_{i}-\widehat{y}_{i}}{y_{i}}\right|,
$$

root mean square error:

$$
\mathrm{RMSE}=\sqrt{\frac{1}{n} \sum_{i=1}^{n}\left(y_{i}-\widehat{y}_{i}\right)^{2}},
$$

Theil's Inequality Coefficient:

$$
\mathrm{TIC}=\frac{\sqrt{\sum_{i=1}^{n}\left(y_{i}-\widehat{y}_{i}\right)^{2}}}{\sqrt{\sum_{i=1}^{n}\left(y_{i}\right)^{2}}+\sqrt{\sum_{i=1}^{n}\left(\hat{y}_{i}\right)^{2}}} .
$$


TABLE 2: Parameters list of RNN obtained from NNCA.

\begin{tabular}{lccccc}
\hline \multicolumn{6}{c}{ Position of hidden layer centers } \\
$c_{1}$ & $c_{2}$ & $c_{3}$ & $c_{4}$ & $c_{5}$ & $c_{6}$ \\
\hline 0.4664 & 0.1387 & 0.7297 & 0.4038 & 0.9834 & 0.9819 \\
0.8050 & 0.2038 & 1.0000 & 0.3347 & 0.8927 & 0.3094 \\
0.6609 & 0.1556 & 0.5499 & 0.9139 & 1.0000 & 0 \\
1.0000 & 0.2958 & 0.0298 & 0.1932 & 0.3967 & 0.6396 \\
0.1667 & 0.3333 & 0.6667 & 0.8333 & 0 & 0.5000 \\
0.6652 & 0.6600 & 0.7054 & 0 & 0.1829 & 0.1522 \\
\hline
\end{tabular}

Width of hidden layer centers

\begin{tabular}{lccccc}
$\sigma_{1}$ & $\sigma_{2}$ & $\sigma_{3}$ & $\sigma_{4}$ & $\sigma_{5}$ & $\sigma_{6}$ \\
\hline 1.0000 & 1.0000 & 1.0000 & 1.0000 & 1.0000 & 1.0000 \\
\hline \multicolumn{5}{c}{ Weights of output layer } \\
$W_{1}$ & $W_{2}$ & $W_{3}$ & $W_{4}$ & $W_{5}$ & $W_{6}$ \\
\hline 0.4340 & 0.5585 & 0.6185 & 0.6064 & 0.6339 & 0.8537 \\
0.5112 & 0.4440 & 0.4670 & 0.3342 & 0.4585 & 0.5194 \\
\hline
\end{tabular}

TABLE 3: Errors of the proposed method, basic PSO-RNN, and conventional RNN (1: strength, 2: structure parameter).

\begin{tabular}{lccccc}
\hline Algorithms & MAE & MRE (\%) & RMSE & TIC & Time (s) \\
\hline Conventional RNN & & & & & \\
1 & 1.1950 & 28.63 & 1.4843 & 0.1675 & \\
2 & 3.6827 & 27.96 & 4.5364 & 0.1452 & 0.9575 \\
$\quad$ Total & 2.4389 & 28.30 & 3.3750 & 0.1470 & \\
\hline Basic PSO-RNN & & & & & \\
1 & 0.4818 & 10.65 & 0.5841 & 0.0690 & \\
2 & 2.0262 & 14.61 & 2.2637 & 0.0766 & 0.7428 \\
$\quad$ Total & 1.2540 & 12.63 & 1.6531 & 0.0761 & \\
\hline Proposed method & & & & & \\
1 & 0.4258 & 9.39 & 0.5157 & 0.0609 & \\
2 & 1.9833 & 14.01 & 2.1177 & 0.0727 & 0.2985 \\
Total & 1.2045 & 11.70 & 1.5412 & 0.0718 & \\
\hline
\end{tabular}

4.2. Properties Prediction. For properties prediction, the production parameters are input, the properties indices are output, $N_{i}$ is 6 , and $N_{o}$ is 2 . Set $r=1$, we obtain $N_{h}=6$ by applying the nearest neighbor-clustering algorithm (NNCA), all the parameters information are list in Table 2.

Based on Table 2, an initial RNN model is developed. We consider the range of initial swarm for both proposed method and basic PSO-RNN by referencing Table 2 . We set $w^{1}=\theta_{1}^{1}=$ $\theta_{2}^{1}=0.5, c_{1 \min }=c_{2 \min }=0.4, c_{1 \max }=c_{2 \max }=0.9, \eta=$ 0.3 , error_goal $=0.01$, maximum iteration $T$ is 5000 , swarm size is 20 for proposed method in this paper, set $w_{\min }=0.4$, $w_{\text {max }}=0.9$ for basic PSO-RNN, and the other parameters of the basic PSO-RNN are agreed with the proposed method.

By employing the proposed GA-IPSO algorithm to optimize the parameters of RNN, we gain the best parameters for the RNN. The training accuracy and the agreement level of the proposed method are shown in Figure 4.

From Figure 4, the prediction values of training dataset have a high level of agreement with the actual data, it can be seen directly that the proposed model has an accuracy
TABLE 4: The parameters value of the RNN obtained from the NNCA.

\begin{tabular}{c}
\hline Position of hidden layer centers \\
$c_{1}$ \\
\hline 0.5453 \\
0.4971 \\
\hline Width of hidden layer centers \\
$\sigma_{1}$ \\
\hline 1.0000 \\
\hline Weights of output layer \\
$W_{1}$ \\
\hline 0.4766 \\
0.5011 \\
0.5117 \\
0.4849 \\
0.4630 \\
0.5397
\end{tabular}

and good tracking performance which reflects the model's outstanding universality.

The model's prediction accuracy also can be reflected from its performance on the test dataset. We show the prediction results of the test dataset of the proposed method, basic PSO-RNN and conventional RNN in Figure 5 to compare their performance. It can be seen obviously that the curve of the proposed GA-IPSO-RNN model is most closed to the actual data curve, which reveals it has the best prediction accuracy and reliability among the three models. The basic PSO-RNN displays worse performance, and the conventional RNN is the worst one with big errors. The detailed numeral comparison of MAE, MRE, RMSE, and TIC is also listed in Table 3.

From data in Table 3, we can see that in terms of MAE, the proposed GA-IPSO-RNN achieves with a decrease of approximately $50.61 \%$ and $3.95 \%$ compared to the other two models, respectively. The same information can be obtained from the other items such as MRE with a decrease of $58.66 \%$ and $7.36 \%$, RMSE with a decrease of $54.33 \%$ and $6.77 \%$, and TIC with a decrease of $51.16 \%$ and $5.65 \%$. Similar results are observed in terms of strength and structure parameter. For strength, MAE with a decrease of $64.37 \%$ and $11.62 \%$, MRE with a decrease of $67.20 \%$ and $11.83 \%$, RMSE with a decrease of $65.26 \%$ and $11.71 \%$, and TIC with a decrease of $63.64 \%$ and $11.74 \%$. For structure parameter, MAE with a decrease of $46.15 \%$ and $2.12 \%$, MRE with a decrease of $49.89 \%$ and $4.11 \%$, RMSE with a decrease of $53.32 \%$ and $6.45 \%$, and TIC with a decrease of $49.93 \%$ and $5.09 \%$. The MAE, MRE, and RMSE of both total and items confirm the prediction accuracy of the proposed method. TIC of both total and items indicates a good level of agreement between the proposed method and the studied process. And the proposed method costs the shortest run time, demonstrates its excellent convergence speed.

4.3. Production Scheme Designer of New Type Carbon Fiber. For production scheme designer, the properties indices are 
TABLE 5: Errors of the proposed method, basic PSO-RNN, and conventional RNN.

\begin{tabular}{|c|c|c|c|c|c|}
\hline Algorithms & MAE & MRE (\%) & RMSE & TIC & Time(s) \\
\hline \multicolumn{6}{|c|}{ Conventional RNN } \\
\hline 1 & 173.1400 & 1876.58 & 341.7052 & 0.9612 & \multirow{7}{*}{1.7296} \\
\hline 2 & 1062.8000 & 1310.66 & 2004.3000 & 0.9391 & \\
\hline 3 & 151.6600 & 816.09 & 285.3062 & 0.9051 & \\
\hline 4 & 171.6800 & 264.56 & 285.1956 & 0.7416 & \\
\hline 5 & 62.6800 & 443.07 & 103.5493 & 0.8054 & \\
\hline 6 & 34.8800 & 533.97 & 63.3625 & 0.9510 & \\
\hline Total & 276.1367 & 874.16 & 847.6972 & 0.9285 & \\
\hline \multicolumn{6}{|c|}{ Basic PSO-RNN } \\
\hline 1 & 1.9652 & 22.73 & 2.2049 & 0.1138 & \multirow{7}{*}{0.4065} \\
\hline 2 & 9.7604 & 11.30 & 10.3417 & 0.0590 & \\
\hline 3 & 2.5932 & 13.19 & 2.7487 & 0.0690 & \\
\hline 4 & 8.9433 & 15.27 & 10.2110 & 0.0806 & \\
\hline 5 & 3.2098 & 20.66 & 3.2757 & 0.1054 & \\
\hline 6 & 0.4920 & 7.38 & 0.6731 & 0.0544 & \\
\hline Total & 4.4940 & 15.09 & 6.2559 & 0.0686 & \\
\hline \multicolumn{6}{|c|}{ Proposed method } \\
\hline 1 & 1.3996 & 14.37 & 1.7597 & 0.1026 & \multirow{7}{*}{0.1464} \\
\hline 2 & 8.2660 & 9.61 & 9.1148 & 0.0518 & \\
\hline 3 & 2.2693 & 11.04 & 2.5107 & 0.0647 & \\
\hline 4 & 8.0976 & 13.47 & 9.1473 & 0.0733 & \\
\hline 5 & 2.9085 & 19.20 & 2.9776 & 0.0944 & \\
\hline 6 & 0.3260 & 4.94 & 0.4054 & 0.0317 & \\
\hline Total & 3.8778 & 12.11 & 5.5555 & 0.0612 & \\
\hline
\end{tabular}

Note: 1: viscosity average molecular weight, 2: conversion ratio, 3: solid content, 4: spinning jet drawing ratio, 5: coagulating bath temperature, 6: total drawing ratio.

input, the production parameters are output, $N_{i}$ is 2 , and $N_{o}$ is 6. Set $r=1$, we obtain $N_{h}=1$ by applying the NNCA. Compared to the properties prediction model, a visual impression of the NNCA can be obtained. For experience formula (6), since the properties prediction model and production scheme design model have the same sum of $N_{i}$ and $N_{o}$, they should almost have the same hidden nodes number; however, they have quite different $N_{h}$ by the NNCA, which indicates its good performance of simplify the RNN structure. And once there adds a new sample, it can be treated online as a training sample for RNN at once, which will improve the prediction accuracy of the model. All the parameters are listed in Table 4.

Based on Table 4, an initial RNN model is developed. We consider the range of initial swarm for both proposed method and basic PSO-RNN by referencing Table 4 . We set $w^{1}=\theta_{1}^{1}=$ $\theta_{2}^{1}=0.5, c_{1 \min }=c_{2 \min }=0.4, c_{1 \max }=c_{2 \max }=0.9, \eta=0.3$, error_goal $=0.01$, maximum iteration $T$ is 5000 , swarm size is 20 for proposed method, set $w_{\text {min }}=0.4, w_{\text {max }}=0.9$ for basic PSO-RNN, and the other parameters of the basic PSORNN are agreed with the proposed method.

By employing the proposed GA-IPSO algorithm to optimize the parameters of the RNN and gain the best parameters. The training accuracy and the agreement level of the proposed method are shown in Figure 6. From Figure 6, the prediction values of training dataset have a high level of agreement with the actual data, it can be seen directly that the proposed model has an accuracy and good tracking performance which reflect the model's outstanding universality.

The model's prediction accuracy can also be reflected from its performance on the test dataset. We show the prediction results of the test dataset of the proposed method, basic PSO-RNN and conventional RNN in Figure 7 to compare their performance. It can be seen obviously that the curve of the proposed GA-IPSO-RNN model is most closed to the actual data curve, which reveals it has the best prediction accuracy and reliability among the three models. The conventional RNN displays the worst performance, and we just compare the proposed method and the basic PSORNN as shown in Figure 8. The detailed comparison of MAE, MRE, RMSE, and TIC is also listed in Table 5.

From data in Table 5, we can see that in terms of MAE, the proposed GA-IPSO-RNN achieve, with a decrease of approximately $98.59 \%$ and $13.71 \%$ compared to the other two models, respectively. The same information can be obtained from the other items such as MRE with a decrease of $98.61 \%$ and $19.75 \%$, RMSE with a decrease of $99.34 \%$ and $11.20 \%$, and TIC with a decrease of $93.41 \%$ and $10.79 \%$. Similar results are observed in terms of viscosity average molecular weight, conversion ratio, solid content, spinning jet drawing ratio, coagulating bath temperature, and total drawing ratio. The MAE, MRE, and RMSE of both total and items confirm 
the prediction accuracy of the proposed method. TIC of both total and items indicates a good level of agreement between the proposed method and the studied process. As the curves shown in Figure 7, the data list in Table 5 also indicates quite unacceptable errors of conventional RNN, and it verifies the proposed method's effective optimization of RNN's parameters indirectly. And the proposed model costs the shortest run time and demonstrates its excellent convergence.

\section{Conclusions}

In this paper, we present a model for using an enhanced RNN to bidirectionally optimize the production process of PAN-based carbon fiber, which can be viewed as properties prediction and new type fiber designer. We adopt the nearest neighbor-clustering algorithm to simplify the structure of RNN and investigate the learning method of the RNN based on the PSO, introduce penalty factor to improve the PSO, then combine GA with the improved PSO to train the RNN. Meanwhile, we compare the GA-IPSO-RNN model to the existing basic PSO-RNN and conventional RNN. The superiority of the proposed model is summarized as below.

(1) Compare with the conventional RNN, the proposed method adopts the nearest neighbor-clustering algorithm to determine the number of hidden notes and provides an effective approach to simplify the network structure. For carbon fiber production process optimization, it can add new samples online, composite all the information, and improve the accuracy.

(2) Based on the same network structure, the proposed GA-IPSO algorithm provides a more efficient search scheme to determine the related parameters of the RNN than the basic PSO.

(3) Testing on the bidirectional optimization of the carbon fiber production process has shown that the proposed method is superior to the basic PSO-RNN and conventional RNN in constructing the network and estimating the outputs.

(4) After the network is constructed, the proposed model can accelerate the convergence speed, decrease iteration time, benefit the ability of getting out of local optimum, increase the capability of the network, then make a quick response, and yield accurate solution during the bidirectional optimization of the carbon fiber production process.

However, the accuracy of the proposed model is still needed to be improved because the training data should be enriched. When the accuracy meets practical production demands, for properties prediction, because production schemes with little difference may lead to the same product properties, so if control parameters changes tiny, we do not need to tune all the parameters or keep them as constants. For production scheme design, after we obtain the scheme by the proposed model, we can alternately use the properties prediction function of the model, prevent cost wasting in practical production directly. This problem should be studied in future work.

\section{Acknowledgments}

This work was supported in part by the Key Project of the National Nature Science Foundation of China (no. 61134009), the National Nature Science Foundation of China (no. 60975059), Specialized Research Fund for Shanghai Leading Talents, Project of the Shanghai Committee of Science and Technology (nos. 11XD1400100 and 11JC1400200), and the Fundamental Research Funds for the Central Universities.

\section{References}

[1] M. S. A. Rahaman, A. F. Ismail, and A. Mustafa, "A review of heat treatment on polyacrylonitrile fiber," Polymer Degradation and Stability, vol. 92, no. 8, pp. 1421-1432, 2007.

[2] N. Yusof and A. F. Ismail, "Post spinning and pyrolysis processes of polyacrylonitrile (PAN)-based carbon fiber and activated carbon fiber: a review," Journal of Analytical and Applied Pyrolysis, vol. 93, pp. 1-13, 2012.

[3] J. Liu, Y. Tian, Y. Chen, J. Liang, L. Zhang, and H. Fong, "A surface treatment technique of electrochemical oxidation to simultaneously improve the interfacial bonding strength and the tensile strength of PAN-based carbon fibers," Materials Chemistry and Physics, vol. 122, no. 2-3, pp. 548-555, 2010.

[4] Y. Wang and W. Yin, "Chemical modification for PAN fibers during heat-treatment process," Physics Procedia, vol. 18, pp. 202-205, 2011.

[5] M. A. Rahman, A. F. Ismail, and A. Mustafa, "The effect of residence time on the physical characteristics of PANbased fibers produced using a solvent-free coagulation process," Materials Science and Engineering A, vol. 448, no. 1-2, pp. 275280, 2007.

[6] X. Liang, Y. S. Ding, L. H. Ren, K. R. Hao, H. P. Wang, and J. J. Chen, "A bio-inspired multi-layered intelligent cooperative controller for stretching process of fiber production," IEEE Transactions on Systems, Man, and Cybernetics C, vol. 42, no. 3, pp. 367-377, 2012.

[7] H. Rennhofer, D. Loidl, S. Puchegger, and H. Peterlik, "Structural development of PAN-based carbon fibers studied by in situ $\mathrm{X}$-ray scattering at high temperatures under load," Carbon, vol. 48, no. 4, pp. 964-971, 2010.

[8] S. S. Belyaev, I. V. Arkhangelsky, and I. V. Makarenko, "Nonisothermal kinetic analysis of oxidative stabilization processes in PAN fibers," Thermochimica Acta, vol. 507-508, pp. 9-14, 2010.

[9] Y. Sugimoto, M. Shioya, K. Yamamoto, and S. Sakurai, "Relationship between axial compression strength and longitudinal microvoid size for PAN-based carbon fibers," Carbon, vol. 50, no. 8, pp. 2860-2869, 2012.

[10] H. E. Kadi, "Modeling the mechanical behavior of fiberreinforced polymeric composite materials using artificial neural networks-a review," Composite Structures, vol. 73, no. 1, pp. 123, 2006.

[11] Y. Yu, C. L. Hui, T. M. Choi, and R. Au, "Intelligent fabric hand prediction system with fuzzy neural network," IEEE Transactions on Systems, Man and Cybernetics C, vol. 40, no. 6, pp. 619-629, 2010. 
[12] D. Du, K. Li, and M. Fei, "A fast multi-output RBF neural network construction method," Neurocomputing, vol. 73, no. 1012, pp. 2196-2202, 2010.

[13] A. Roy, S. Govil, and R. Miranda, "A neural-network learning theory and a polynomial time RBF algorithm," IEEE Transactions on Neural Networks, vol. 8, no. 6, pp. 1301-1313, 1997.

[14] X. Hong and S. Chen, "A new RBF neural network with boundary value constraints," IEEE Transactions on Systems, Man, and Cybernetics B, vol. 39, no. 1, pp. 298-303, 2009.

[15] G. B. Huang, P. Saratchandran, and N. Sundararajan, "A generalized growing and pruning RBF (GGAP-RBF) neural network for function approximation," IEEE Transactions on Neural Networks, vol. 16, no. 1, pp. 57-67, 2005.

[16] J.-F. Qiao and H.-G. Han, "Identification and modeling of nonlinear dynamical systems using a novel self-organizing RBF-based approach," Automatica, vol. 48, no. 8, pp. 1729-1734, 2012.

[17] Y. Wang and G. Liu, "A forecasting method based on online self-correcting single model RBF neural network," Procedia Engineering, vol. 29, pp. 2516-2520, 2012.

[18] Y. F. Hu, Y. S. Ding, and K. R. Hao, "An immune cooperative particle swarm optimization algorithm for fault-tolerant routing optimization in heterogeneous wireless sensor networks," Mathematical Problems in Engineering, vol. 2012, Article ID 743728, 19 pages, 2012.

[19] A. Alfi, "PSO with adaptive mutation and inertia weight and its application in parameter estimation of dynamic systems," Acta Automatica Sinica, vol. 37, no. 5, pp. 541-549, 2011.

[20] M. A. Behrang, E. Assareh, A. R. Noghrehabadi, and A. Ghanbarzadeh, "New sunshine-based models for predicting global solar radiation using PSO (particle swarm optimization) technique," Energy, vol. 36, no. 5, pp. 3036-3049, 2011.

[21] A. Mahor and S. Rangnekar, "Short term generation scheduling of cascaded hydro electric system using novel self adaptive inertia weight PSO," International Journal of Electrical Power and Energy Systems, vol. 34, no. 1, pp. 1-9, 2012.

[22] Y. Tang, H. Gao, J. Kurths, and J. Fang, "Evolutionary pinning control and its application in UAV coordination," IEEE Transactions on Industrial Informatics, vol. 8, no. 4, pp. 828-838, 2012.

[23] B. Luitel and G. K. Venayagamoorthy, "Quantum inspired PSO for the optimization of simultaneous recurrent neural networks as MIMO learning systems," Neural Networks, vol. 23, no. 5, pp. 583-586, 2010.

[24] B. Vasumathi and S. Moorthi, "Implementation of hybrid ANNPSO algorithm on FPGA for harmonic estimation," Engineering Applications of Artificial Intelligence, vol. 25, no. 3, pp. 476-483, 2012.

[25] S. K. Oh, W. D. Kim, W. Pedrycz, and B. J. Park, "Polynomialbased radial basis function neural networks (P-RBF NNs) realized with the aid of particle swarm optimization," Fuzzy Sets and Systems, vol. 163, no. 1, pp. 54-77, 2011.

[26] C. M. Huang and F. L. Wang, "An RBF network with OLS and EPSO algorithms for real-time power dispatch," IEEE Transactions on Power Systems, vol. 22, no. 1, pp. 96-104, 2007.

[27] J. Li and X. Liu, "Melt index prediction by RBF neural network optimized with an MPSO-SA hybrid algorithm," Neurocomputing, vol. 74, no. 5, pp. 735-740, 2011.

[28] J. Moody and C. J. Darken, "Fast learning in networks of locallyturned processing units," Neural Computation, vol. 1, no. 2, pp. 281-294, 1989.
[29] R. A. Jarvis and E. A. Patrick, "Clustering using a similarity measure based on shared near neighbors," IEEE Transactions on Computers, vol. C-22, no. 11, pp. 1025-1034, 1973.

[30] J. Kennedy and R. Eberhart, "Particle swarm optimization," in Proceedings of the 4th IEEE International Conference on Neural Networks, pp. 1942-1948, December 1995.

[31] Z. Ditzian, "Relating smoothness to expressions involving Fourier coefficients or to a Fourier transform," Journal of Approximation Theory, vol. 164, no. 10, pp. 1369-1389, 2012. 


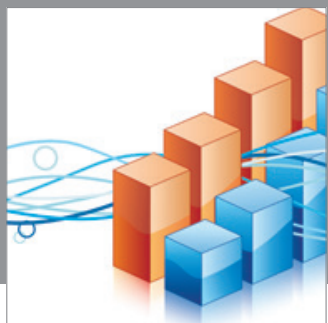

Advances in

Operations Research

mansans

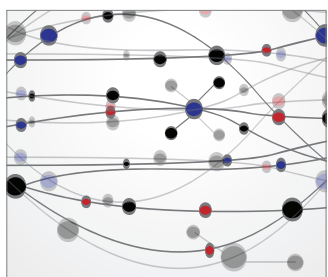

The Scientific World Journal
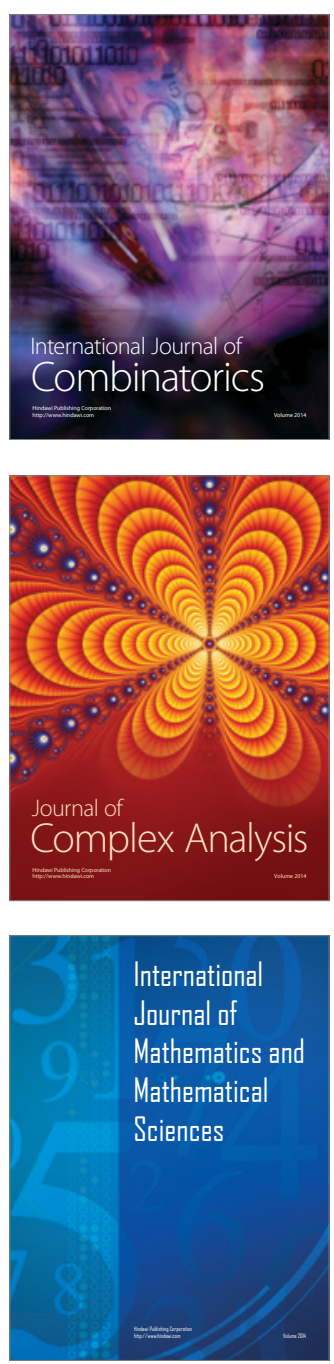
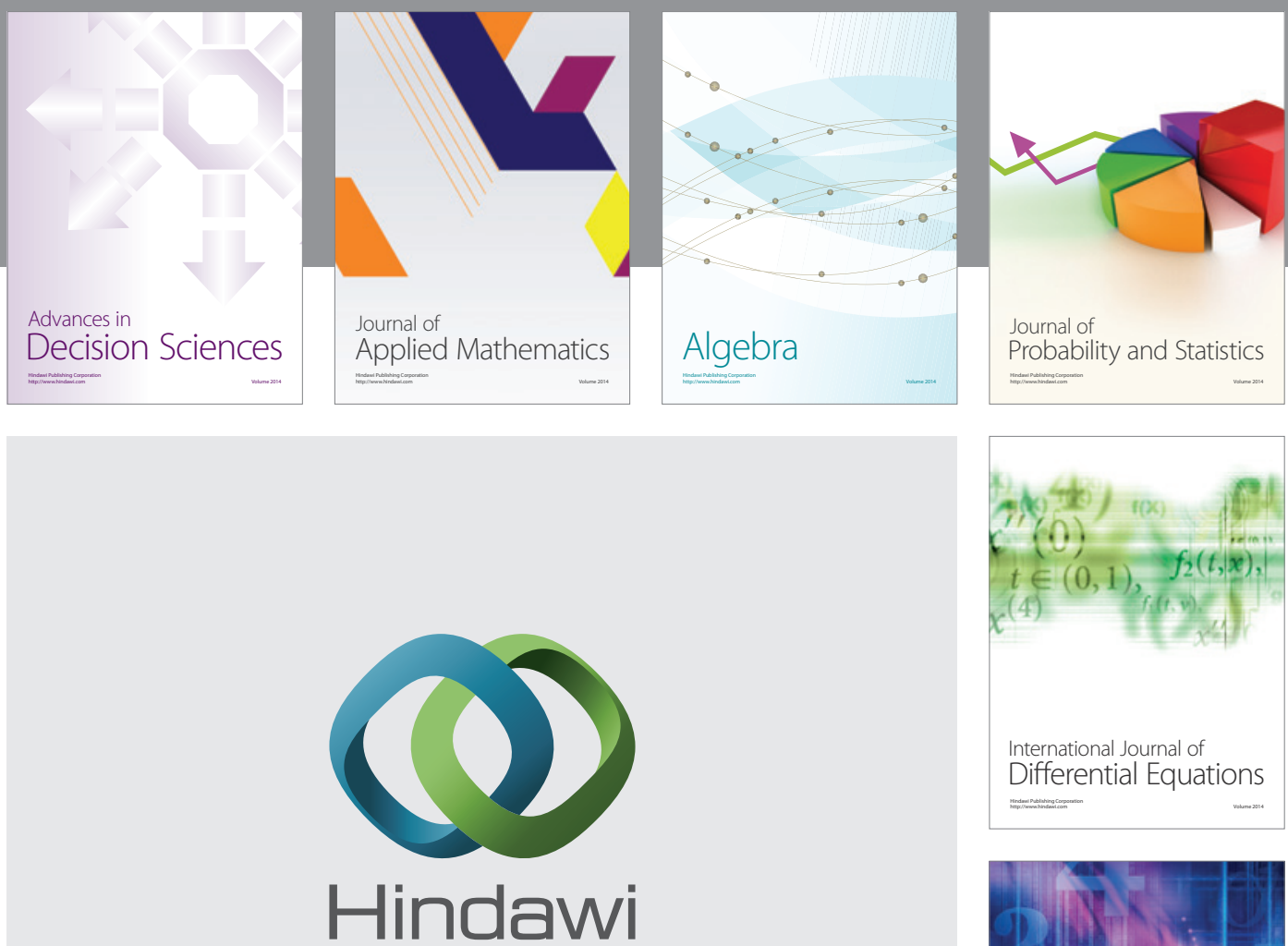

Submit your manuscripts at http://www.hindawi.com
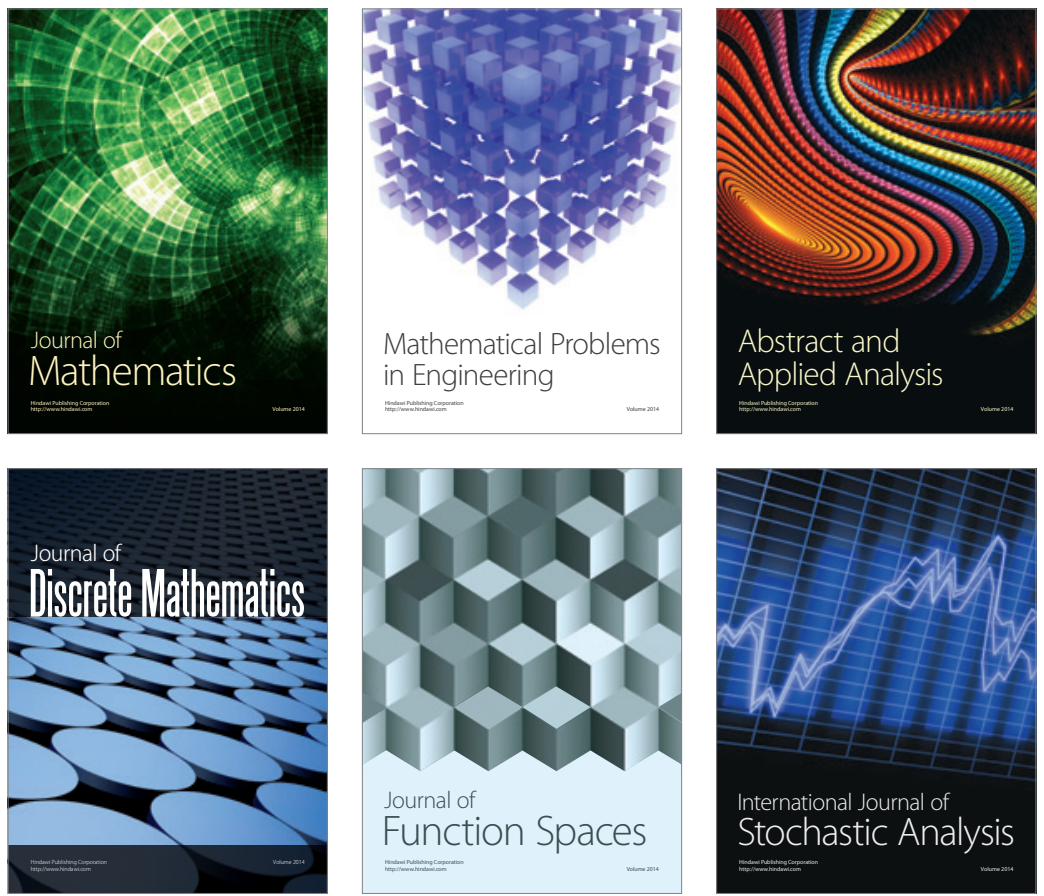

Journal of

Function Spaces

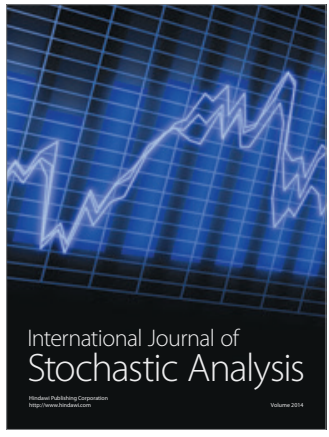

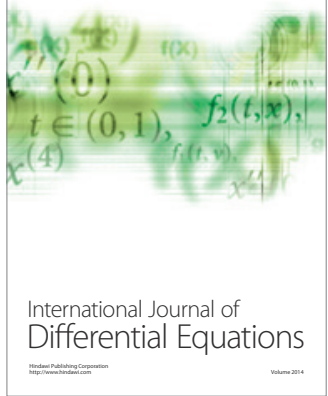
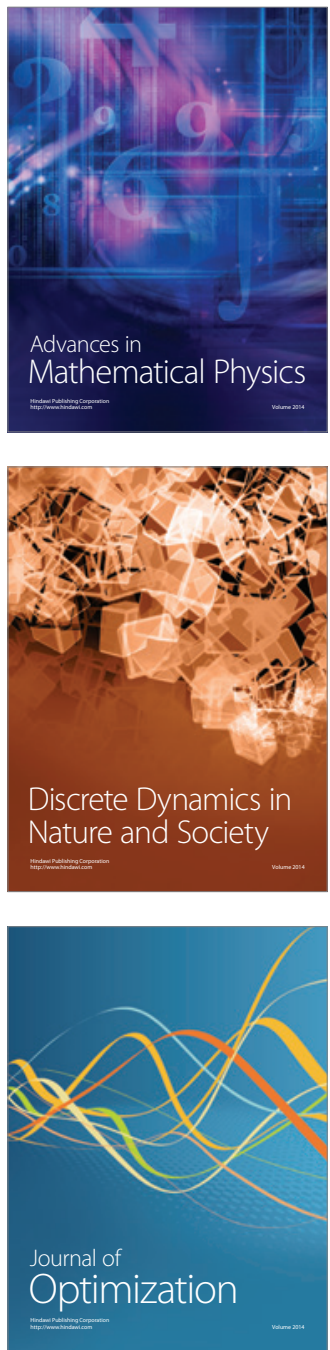